\title{
Kinetic Approach to Model Reactive Transport and Mixed Salt Precipitation in a Coupled Free-Flow-Porous-Media System
}

\author{
V. A. Jambhekar ${ }^{1}$ - E. Mejri ${ }^{1,3}$ - N. Schröder ${ }^{1}$. \\ R. Helmig ${ }^{1}$ - N. Shokri
}

Received: 21 April 2015 / Accepted: 28 February 2016 / Published online: 15 March 2016

(C) Springer Science+Business Media Dordrecht 2016

\begin{abstract}
Evaporative salinization of the agricultural soil is a chronic problem in arid, semiarid and coastal regions worldwide. As a shallow subsurface issue, it is strongly influenced by the free-flow (henceforth, FF) and porous-media (henceforth, PM) flow and transport processes. In specific, it is mainly affected by the mass, momentum and energy exchange between the FF and the PM regions. Furthermore, salt precipitation in such systems is strongly determined by the interaction between different dissolved ionic species. In the scope of this work, we extend the REV-scale coupled FF-PM model concept for salinization proposed by Jambhekar et al. (Transp Porous Media, 2015), to describe reactive transport of ionic species $\mathrm{Na}^{+}, \mathrm{Cl}^{-}$and $\mathrm{I}^{-}$and mixed salt precipitation (here, $\mathrm{NaCl}$ and $\mathrm{NaI}$ ). In the first part of our numerical analysis, we illustrate precipitation behavior of $\mathrm{NaCl}$ using equilibrium and kinetic approaches. Both approaches are found to be in good agreement with experimental observations. However, in the literature it is often discussed that for mixed salt systems found in the nature, the equilibrium precipitation-dissolution assumption is not easily justifiable. Therefore, in the second part of our numerical analysis, we extend the kinetic approach to
\end{abstract}

V. A. Jambhekar

vishaljambhekar@gmail.com

E. Mejri

Emna.Mejri@iws.uni-stuttgart.de

N. Schröder

Natalie.Schroeder@iws.uni-stuttgart.de

R. Helmig

Rainer.Helmig@iws.uni-stuttgart.de

N. Shokri

nima.shokri@manchester.ac.uk

1 Department of Hydromechanics and Modelling of Hydrosystems, University of Stuttgart, Stuttgart, Germany

2 School of Chemical Engineering and Analytical Science, The University of Manchester, Manchester, UK

3 Laboratoire de Modelisation en Hydraulique et Environnement, National Engineering School of Tunis, University Tunis el Manar, Tunis, Tunisia 
describe mixed salt precipitation in an $\mathrm{NaCl}-\mathrm{NaI}$ system and compare it with the equilibrium precipitation approach. Our numerical analysis indicate that the simulation results for these approaches are very similar and analogous with the phenomenological explanations.

Keywords Reactive transport · Precipitation · Dissolution · Coupling · Evaporation

\section{Introduction}

Soil salinization is one of the most serious agricultural problems, predominantly observed in arid, semiarid and coastal areas. It refers to accumulation of salt in the pores in the shallow subsurface or at the soil-atmosphere interface. According to Munns (2011), around $3 \%$ of the world's land surface (approximately 351.5 Mha) has been rendered useless by the salinization problem.

The primary cause of the agricultural salinization is evaporative reduction of the irrigated water volume and resulting salt precipitation, as shown schematically in Fig. 1. The irrigation water normally taken from sources such as rivers, lakes or water reservoirs always contains some amount of dissolved salts. Thus, salinization to a certain extent is inevitable. However, encouraged excessive supplementary irrigation practices for ambitious crop yield and poor natural drainage of the irrigated fields risk acute soil salinization and therefore reduction in the crop yield in the long run. Therefore, in the last decades there is a developing research interest to understand the physical processes related to evaporation-driven soil salinization in the shallow subsurface.

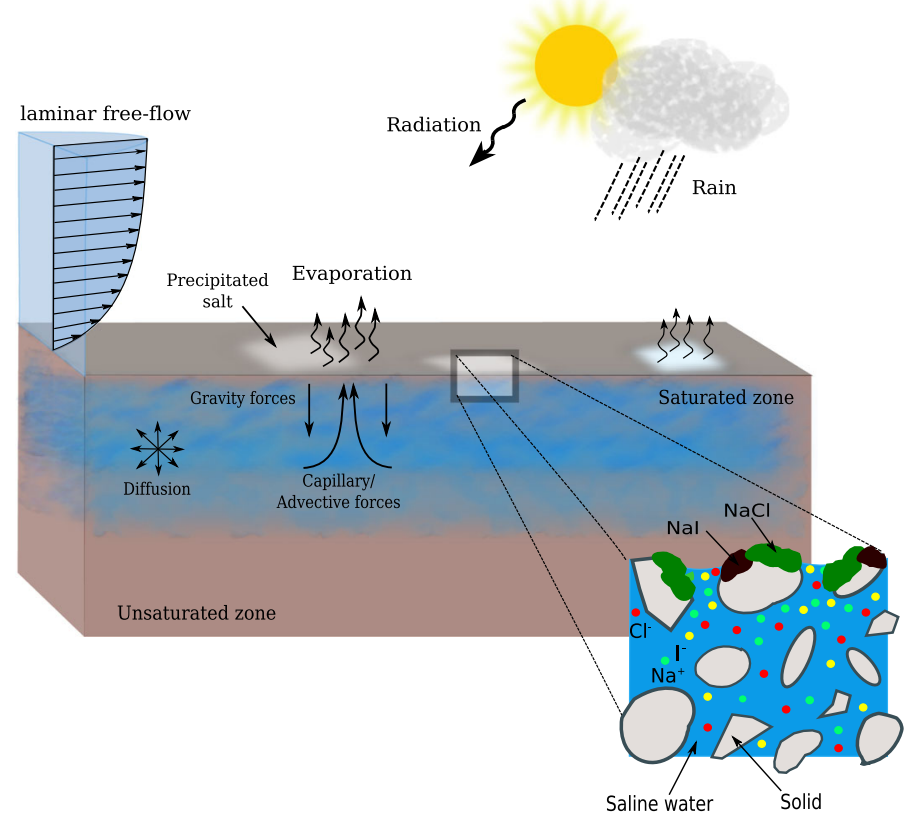

Fig. 1 Relevant interface processes for evaporative salinization: FF velocity, solar radiation, rainfall events, evaporative water loss, salt transport and precipitation and exchange fluxes at the FF-PM interface are illustrated 
As depicted in Fig. 1, during evaporative salinization dissolved salt transport is significantly influenced by the flow and transport processes in the PM and the FF. On the PM side dissolved salt transport is governed by capillary, viscous and gravitational forces and advective and diffusive processes. On the FF side, wind speed, temperature, humidity and solar radiation control evaporation strongly and therefore influence soil salinization.

For FF-PM interaction, evaporation takes place either directly in the water-filled pores at the FF-PM interface (saturated case) or within the PM (unsaturated case), where the water vapor is transported to the FF by diffusion through the PM gas phase. Evaporation promotes salt accumulation and precipitation at the evaporation sites, which has the following implications: (a) decrease in the saturation vapor pressure of saline water due to an increase in the osmotic potential (salt concentration) at the evaporation site (Battistelli et al. 1997; Kelly and Selker 2001; Nachshon et al. 2011a) and (b) evolution of the pore geometry due to salt precipitation at the evaporation site. Therefore, evaporative salinization can influence the evaporation dynamics through change of the void space by salt precipitation and vapor pressure lowering.

Numerical modeling of saline water evaporation from a naturally existing PM system in contact with the FF is hardly addressed in the literature. In our previous work Jambhekar et al. (2015), we have extended the model concept proposed by Mosthaf et al. (2011) to describe evaporative salinization in a coupled non-isothermal compositional FF-PM system. In Jambhekar et al. (2015) we used a simplified or Layman's approach to describe precipitation of a single dissolved salt $(\mathrm{NaCl})$, considered to be one component.

However, in a natural shallow subsurface system (unsaturated zone), dissolved salts exist in the form of dissociated ionic species, e.g., $\mathrm{Na}^{+}, \mathrm{Cl}^{-}$and $\mathrm{I}^{-}$(Fig. 1). In such a system, one needs to describe reactive transport of individual ionic species. In addition, interactions between ions of different species and its influence on the precipitation-dissolution dynamics of various salts (e.g., $\mathrm{NaCl}$ and $\mathrm{NaI}$ in Fig. 1) must be accounted. Moreover, complexity of such systems may increase dramatically in the presence of flow and transport processes (Steefel and Cappellen 1990).

In the context of chemical complexity, a cation (e.g., $\mathrm{Na}^{+}$) present in the liquid phase can combine with any existing potential anions (e.g., $\mathrm{Cl}^{-}$or $\mathrm{I}^{-}$). This implies that, at any given instance, depending on inter-ionic interaction and solution-solid-salt interaction precipitation or dissolution can take place in the system. For such systems, Crowe and Langstaffe (1987), Steefel and Cappellen (1990) and Mayer et al. (2002) recommended the use of a generalized kinetic approach to model precipitation-dissolution processes, as the assumption of equilibrium is not easily justifiable.

In this work, we present a new robust representative elementary volume (REV) scale approach for reactive transport and precipitation-dissolution in a drying PM system coupled with the FF. For reactive transport and precipitation, the transition from microscale to REV scale is schematically shown in Fig. 2. The new approach also accounts for interactions between different dissolved ionic species and mineral precipitates.

\section{Objectives and Structure}

In this manuscript, we are addressing the following objectives:

(1) Development and implementation of an REV-scale approach for reactive transport and rate-controlled kinetic precipitation-dissolution processes. 


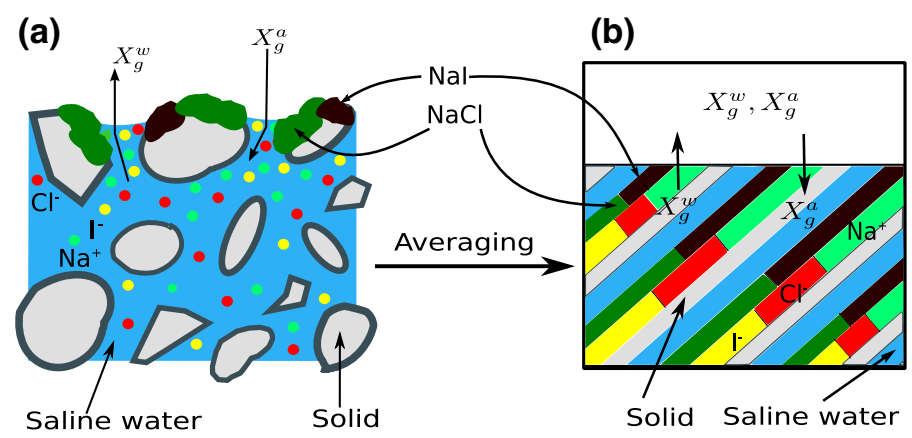

Fig. 2 Evaporation-driven transport and precipitation-dissolution reaction processes for dissolved ionic species, namely $\mathrm{Na}^{+}, \mathrm{Cl}^{-}$and $\mathrm{I}^{-}$. Related to the evaporation-driven transport higher ionic concentrations are observed at the water-air interface. Salt precipitation is observed at the fluid-solid interface as the liquid-phase ionic saturation reaches its maximum. On the REV scale, only volume averaged spatial distribution is available for dissolved ionic species and different fluid and solid phases. a Microscale. b Macroscale (REV)

(2) Numerical modeling of evaporation-driven reactive transport of $\mathrm{Na}^{+}, \mathrm{Cl}^{-}$and precipitation of $\mathrm{NaCl}$. The simulation results will be compared with the experimental data presented by Rad Norouzi et al. (2013).

(3) Calibration of the kinetic precipitation approach in the model against the experimental data.

(4) Analysis of the precipitation dynamics of $\mathrm{NaCl}$ and $\mathrm{NaI}$ for brine-containing ionic species $\mathrm{Na}^{+}, \mathrm{Cl}^{-}$and $\mathrm{I}^{-}$with the new kinetic precipitation model.

The paper is structured as follows: In Sect. 3, we discuss the reactive salt precipitation approach. In Sect. 4, we present the model concept for evaporative salinization, namely FF model, PM model and coupling conditions. In Sect. 5, we briefly address the primary variables and the computational algorithm. The experimental and simulation setup are described in Sects. 6 and 7, respectively. Numerical experiments, calibration against the experimental data and different precipitation situations are given in Sect. 8. Final remarks and outlook are summarized in Sect. 9.

\section{Reactive Precipitation Approach}

Reaction taking place within a single phase (e.g., liquid phase) is termed as homogeneous reaction, and reaction taking place between components from different phases (e.g., solid and liquid phase) is termed heterogeneous reaction (Domenico and Schwartz 1990; Appelo and Postma 2005). The precipitation-dissolution process of salt (e.g., $\mathrm{NaCl}$ ) is a heterogeneous reaction

$$
\mathrm{NaCl} \rightleftharpoons \mathrm{Na}^{+}+\mathrm{Cl}^{-} .
$$

In the following, for mixed salt scenario, we will also be looking at the precipitationdissolution processes of $\mathrm{NaI}$

$$
\mathrm{NaI} \rightleftharpoons \mathrm{Na}^{+}+\mathrm{I}^{-}
$$

A reactive PM flow system is mainly driven by the feedback between precipitated salt and surrounding local composition of the fluid phase. As a feedback to variation of the fluid 


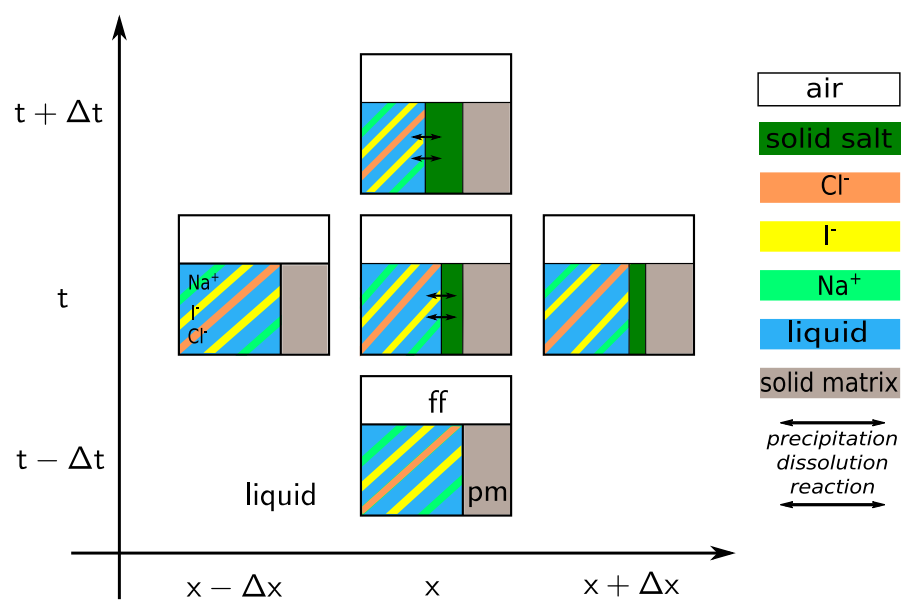

Fig. 3 An REV-scale schematic representation of spatial and temporal distribution of reactive precipitationdissolution processes (for ionic species $\mathrm{Na}^{+}, \mathrm{Cl}^{-}$and ${ }^{-}$). Salt precipitate distribution can be seen in space and time. Bidirectional arrows indicate precipitation-dissolution processes

composition, solid salt aggregation is regulated through precipitation-dissolution processes (Steefel and Cappellen 1990). However, salt aggregation in a PM system may vary in space and time as illustrated schematically in Fig. 3. Here, at a given time $(t)$, differing salt aggregates (shown in green) persist at different locations in the PM system, and in addition to this, solid salt present at any location (x) may vary with time. Therefore, the reaction taking place at a given location can potentially influence the reaction elsewhere in the flow field (Domenico and Schwartz 1990).

\subsection{Ionic Activity}

The activity of the dissolved ionic species indicates the deviation of the solution from ideal behavior. The non-idealities in a solution are mainly caused by the electrostatic interactions between the ionic species. For a given species $i$, the ionic activity [ $i]$ (also called as thermodynamically effective concentration) can be related to its molality as Domenico and Schwartz (1990)

$$
[i]=\gamma_{i} \frac{m_{i}}{m_{0}}
$$

where $\gamma_{i}(-)$ is the ionic activity coefficient, $m_{i} \mathrm{~mol} / \mathrm{kg}$ is the molality (henceforth, $\mathrm{M}$ ) of component $i$, and $m_{0}$ is the standard state molality of $1.0 \mathrm{~mol} / \mathrm{kg}$. Here, the standard state molality is used to make the equation dimensionless. For dilute solutions, $\gamma_{i}$ is close to unity and decreases with salinity. For higher salinities, the activity coefficient shows ascending behavior in many cases, where it may also exceed unity (see Fig. 4). Detailed discussion on the commonly used activity models is given in Domenico and Schwartz (1990) and Appelo and Postma (2005). For example, the Debye-Hückle model is the simplest

$$
\log \gamma_{i}=-A z_{i}^{2} \sqrt{I}
$$


Fig. 4 Activity coefficient $(\gamma)$ for ionic species (subscript $i$ ) plotted over the ionic strength. Solid lines correspond to the activity coefficients determined using the Truesdell and Jones model (see Eq. 7), and that calculated using the Debye-Hückle model are represented by symbols (see Eq. 6)

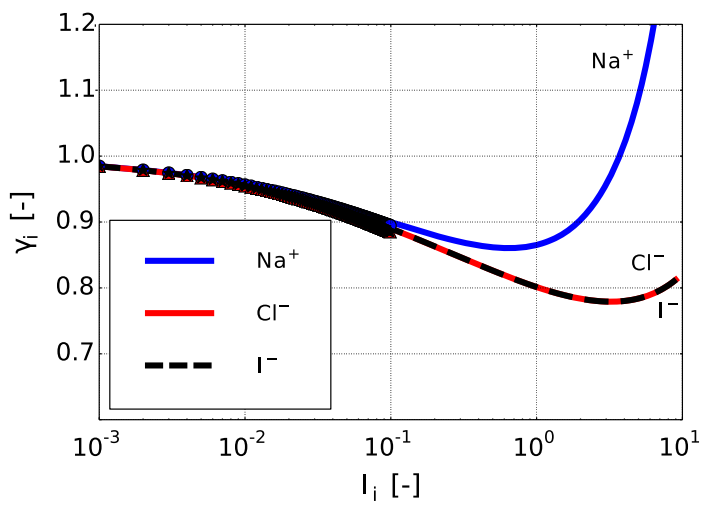

Table 1 Activity coefficient parameters for existing ions for Debye-Hückle model and the Truesdell and Jhones model (Appelo and Postma 2005)

\begin{tabular}{|c|c|c|c|c|c|c|}
\hline Activity model & Ion & \multicolumn{5}{|c|}{ parameters } \\
\hline \multirow[b]{2}{*}{ Debye-Hückle } & \multirow[b]{2}{*}{$\begin{array}{c}\mathrm{Na}^{+} \\
\mathrm{Cl}^{-} \\
\mathrm{I}^{-} \\
\end{array}$} & \multicolumn{2}{|c|}{$\stackrel{\circ}{a}_{i} \times 10^{-10}[\mathrm{~m}]$} & $A[-]$ & $B \times 10^{-10}$ & $\frac{1}{m}$ \\
\hline & & \multicolumn{2}{|l|}{$\begin{array}{c}4.0-4.5 \\
3.0\end{array}$} & \multirow{3}{*}{0.5085} & \multirow{3}{*}{\multicolumn{2}{|c|}{0.3285}} \\
\hline \multirow[b]{2}{*}{ Truesdell and Jones } & \multirow[b]{2}{*}{$\begin{array}{c}\mathrm{Na}^{+} \\
\mathrm{Cl}^{-} \\
\mathrm{I}^{-}\end{array}$} & $a_{i} \times 10^{-10}[\mathrm{~m}]$ & $b_{i}[-]$ & & & \\
\hline & & $\begin{array}{l}4.0 \\
3.5\end{array}$ & $\begin{array}{l}0.075 \\
0.015\end{array}$ & & & \\
\hline
\end{tabular}

where the constant $A(-)$ is temperature dependent, $z_{i}$ is the ionic charge, and the ionic strength $I(-)$ of the solution is given as

$$
I=\frac{1}{2} \sum_{i} \frac{m_{i}}{m_{0}} z_{i}^{2} .
$$

The ionic strength of a solution is a measure of the concentration of ions in that solution. The Debye-Hückle model is only applicable up to ionic strength 0.005 . The modified version of the Debye-Hückle equation which is applicable up to ionic strength of 0.1 is given as

$$
\log \gamma_{i}=\frac{-A z_{i}^{2} \sqrt{I}}{1+B a_{i}^{\circ} \sqrt{I}},
$$

where $A(-)$ and $B 1 / \mathrm{m}$ are temperature-dependent constants and $a_{i} \mathrm{~m}$ is the radius of the ion (Domenico and Schwartz 1990). As discussed in Appelo and Postma (2005), for higher salinity an additional fitting term to Eq. 6 is introduced by Truesdell and Jones (1973)

$$
\log \gamma_{i}=\frac{-A z_{i}^{2} \sqrt{I}}{1+B a_{i} \sqrt{I}}+b_{i} I,
$$

where $a_{i}$ and $b_{i}$ are the ion-specific fitting parameter as given in Table 1 . The Truesdell and Jones activity model (see Eq. 7) gives reasonable approximations up to ionic strength $I \leq 2$. 
Variation of the activity coefficients $\left(\gamma_{i}\right)$ for different ionic species involved in the scope of this work over the ionic strength is presented in Fig. 4. Here, the activity coefficients determined using the Truesdell and Jones model are represented by solid lines and the symbols correspond to the extended Debye-Hückle model. The extended Debye-Hückle model is well in accordance with the Truesdell and Jones model (Appelo and Postma 2005) within the applicability limit. Here, it is important to notice that the activity behavior for $\mathrm{Cl}^{-}$and $\mathrm{I}^{-}$are identical. Moreover, one must also note that, even if the Truesdell and Jones model is accurate up to $I \leq 2$, it is largely used for geochemical applications where ionic strength $I$ can also be $>10.0$. Therefore, Fig. 4 depicts activity behavior up to $I \leq 10$.

In addition to above models, activity models like the Devies model (Appelo and Postma 2005) and the Pitzer and Kim model (1974) are also available. However, the Devies model is only applicable to the ionic strength of 0.5 , and the Pitzer and Kim model is limited in terms of components that can be treated (Crowe and Langstaffe 1987). Moreover, ion paring values needed for the Pitzer and Kim formulation are not available at hand and very difficult to find for a multi-component system. Thus, in line with most of the geochemical codes (e.g., TOUGH2 Xu et al. 2012), we use the Truesdell and Jones model.

\subsection{Law of Mass Action}

The law of mass action is a widely used mathematical model that explains and predicts the equilibrium behavior of a reversible chemical reaction. For example, for a generalized chemical reaction

$$
A^{p}+B^{q}=X^{r}+Y^{s}
$$

the equilibrium constant $K_{\text {eq }}$ reac $(-)$ is given as

$$
K_{\mathrm{eq}}=\frac{[\mathrm{A}]^{p}[\mathrm{~B}]^{q}}{[\mathrm{X}]^{r}[\mathrm{Y}]^{s}},
$$

where $p, q, r$ and $s$ are stoichiometric coefficients of species $A, B, X$ and $Y$, respectively. The equilibrium constant is normally a function of temperature and to a smaller extent of pressure. Thus, for the chemical reactions given by Eq. 1, the equilibrium constant $K_{\mathrm{NaCl}_{\mathrm{eq}}}$ $(-)$ is given as

$$
K_{\mathrm{eq}_{\mathrm{NaCl}}}=\frac{\left[\mathrm{Na}^{+}\right]\left[\mathrm{Cl}^{-}\right]}{[\mathrm{NaCl}]},
$$

Here, activity of the precipitated solid salt $[\mathrm{NaCl}]=1.0$ and the equilibrium constant $K_{\mathrm{NaCl}_{\text {eq }}}$ is 28.092 (-). Similar to $K_{\mathrm{NaCl}_{\text {eq }}}$, equilibrium constant $K_{\mathrm{NaI}_{\mathrm{eq}}}=126.667$ (-) is also determined for the reaction given by Eq. 2 .

\subsection{Equilibrium Versus Kinetic Reaction}

The state of chemical equilibrium of a saline PM system describes its condition of maximum thermodynamic stability. At this state, there is no potential to alter the mass distribution between dissolved and precipitated salts. As the system moves away from this state, with the available chemical potential, it continuously attempts to attend chemical equilibrium through precipitation-dissolution processes.

The existence of equilibrium in such PM flow system is audited by the competition between the reaction and the transport processes (Appelo and Postma 2005). If the reactive processes are faster in relation to the species transport, local chemical equilibrium exists. Adversely, 


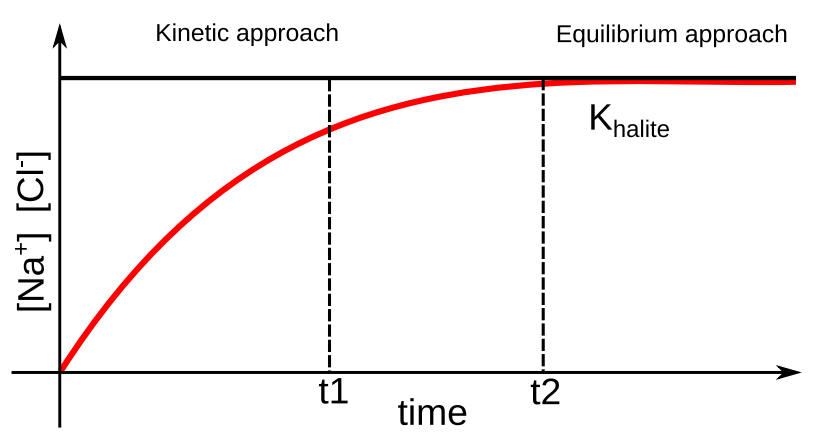

Fig. 5 Halite $(\mathrm{NaCl})$ dissolution in pure water is schematically shown by the red line. The black line represents equilibrium assumption. Here, for the salt dissolution processes, equilibrium state is reached at time $t 2$, and thus, prior to $t 2$, the dissolution processes should be modeled using kinetic approach. Moreover, being a generalized approach it can also be used for modeling complete dissolution processes (Appelo and Postma 2005)

if the reaction processes are slower than the species transport, chemical non-equilibrium persists locally. Here, a kinetic formulation must be adopted for the reaction description (Domenico and Schwartz 1990).

The timescale for a chemical reaction can be related to the time scale for transport using the Dammköhler number $(D a)$. It is defined as the ratio of the characteristic reaction rate to the transport rate. It can also be expressed as the ratio of transport time to the reaction time

$$
D a=\frac{\dot{m}_{\mathrm{R}}}{\dot{m}_{\mathrm{T}}}=\frac{\tau_{\mathrm{T}}}{\tau_{\mathrm{R}}} .
$$

Here, the subscript $\mathrm{T}$ and $\mathrm{R}$ stand for transport and reaction, respectively. Based on the mode of transport (i.e., advection or diffusion), the Dammköhler number can be differentiated. If $\dot{m}_{\mathrm{T}}<\dot{m}_{\mathrm{R}}$, the equilibrium model can be used, otherwise, a non-equilibrium (kinetic) approach is needed.

\subsection{Deviation from Equilibrium State}

Literature review shows that the equilibrium assumption is widely used for modeling mineral precipitation-dissolution processes. However, as discussed by Appelo and Postma (2005), even for a simple situation of $\mathrm{NaCl}$ dissolution in pure water given by Eq. 1, the dissolution reaction proceeds from left to right so long as equilibrium is not built. The first existence of equilibrium is shown schematically by time $t_{2}$ in Fig. 5 . Therefore, the complete processes can not be studied using the equilibrium point of view. Here, a kinetic description of the dissolution processes is needed till time $t_{2}$.

Moreover, for mixed salt systems found in the nature, interactions between different ionic species play a significant role in the salt precipitation dynamics. These ionic interactions can also potentially influence the precipitation of other salts in the system (Crowe and Langstaffe 1987). Furthermore, the precipitation-dissolution behavior of distinct salts present in such systems vary from each other. Therefore, for a mixed salt system, the equilibrium reaction assumption is not easily justifiable.

According to Crowe and Langstaffe (1987), Steefel and Cappellen (1990), Mayer et al. (2002) and Appelo and Postma (2005), in such geochemical systems the precipitationdissolution reactions are usually assumed to be kinetically controlled, and the equilibrium 

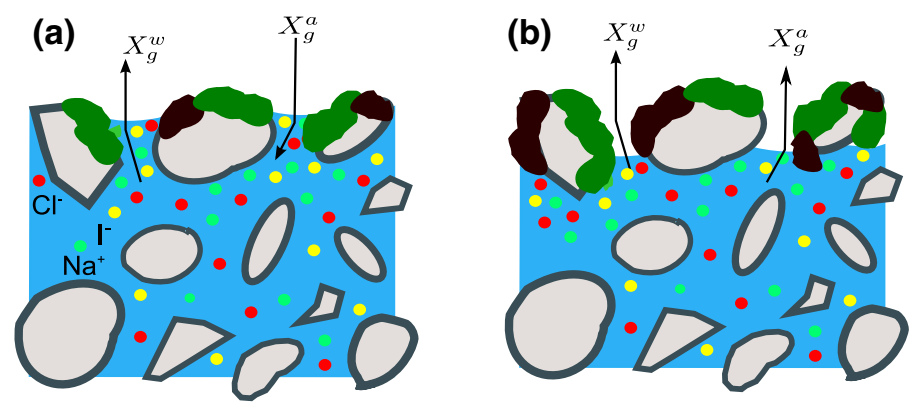

Fig. 6 a Interfacial surface area between solid and liquid $\left(A_{n}\right)$ depends on both pore space occupied by precipitated salt $\left(\phi_{\mathrm{S}}\right)$ and liquid-phase saturation $\left(S_{w}\right)$ (see Eq. 13). b The interfacial area between solid and liquid decreases with saturation during the course of evaporation

concept is applied only if the reaction processes are significantly rapid ( $\tau_{\mathrm{R}} \ll \tau_{\mathrm{T}}$ ). In the scope of this work, we extend the kinetically controlled precipitation-dissolution approach discussed by Lasaga et al. (1994), Appelo and Postma (2005) and Xu et al. (2012) to describe mixed salt precipitation and dissolution processes in the shallow subsurface. Our new model is given as

$$
r_{n}= \pm \underbrace{k_{n} A_{n}\left(S_{w}\right)}_{k_{\text {eff }}}\left|1-\Omega_{n}^{\theta}\right|^{\eta} \quad n=1 \ldots N_{q},
$$

where $n$ is the reacting mineral index. Positive values of $r_{n}$ indicated dissolution and the negative values precipitation, and $k_{n} \mathrm{~mol} / \mathrm{m}^{2} \mathrm{~s}$ is the precipitation or dissolution rate. The fitting parameters $\eta$ and $\theta$ are determined using experiments; not always, but usually, they are equal to unity $\mathrm{Xu}$ et al. (2012). Here, $A\left(S_{w}\right) \mathrm{m}^{2} / \mathrm{m}^{3}$ is the solid-liquid interfacial area and $\Omega_{n}$ is the kinetic saturation state.

For precipitation-dissolution reactions, reactive surface area between the solid and the liquid phase, as shown in Fig. 6, is a crucial parameter controlling the overall rate of reaction. Therefore, an appropriate constitutive relationship should be considered for its description (Gamazo et al. 2012; Steefel and Cappellen 1990). The constitutive relation used in the scope of this work is given as Clement et al. (1996)

$$
A_{n}=500\left(1-\frac{\phi_{\mathrm{S}}}{\phi_{0}}\right)^{2 / 3} S_{w}
$$

Here, $\phi_{\mathrm{S}}$ is pore volume occupied by the precipitated salt, and $\phi_{0}$ is the initial porosity. During evaporation, as the PM dries out, the available solid-liquid interfacial area will be reduced. Therefore, based on phenomenological explanations, the interfacial area is weighted with the liquid-phase saturation $\left(S_{w}\right)$ (see Eq. 13). It is important to note that the factor 500 represents the specific solid surface of the porous medium and it strongly depends on the particle size distribution in the porous medium.

The deviation from equilibrium state is determined by the kinetic saturation state of the solution $\Omega_{n}$

$$
\Omega_{n}=K_{n_{\mathrm{eq}}}^{-1} \prod_{j=1}^{N_{c}} \gamma_{j} \frac{m_{j}}{m_{0}} \quad n=1 \ldots N_{p}
$$


where $K_{n_{\mathrm{eq}}}$ is the corresponding equilibrium constant, $n$ is the reacting mineral index, and $j$ is the dissolved ion species index. Equation 14 can also be written as

$$
\Omega_{n}=\frac{K_{\mathrm{sp}_{n}}}{K_{\mathrm{eq}_{n}}} \quad n=1 \ldots N_{p},
$$

where $K_{\mathrm{sp}_{n}}$ is the solubility product for mineral index $n$. Equations 14 and 15 are valid for reactions involving monovalent ions (i.e., stoichiometric coefficients $=1$ ), involved in this reaction. For generalized formulation we refer to Appelo and Postma (2005) and Domenico and Schwartz (1990) (see Eq. 9). Here, the kinetic saturation state $\left(\Omega_{n}\right)$ has the following possible consequences on the reaction process

(1) If the $K_{\mathrm{sp}_{n}}>K_{\mathrm{eq}_{n}}$ (i.e., $\Omega_{n}>1.0$ ), the reaction progresses from right to left (i.e., $\mathrm{NaCl}$ precipitates).

(2) If the $K_{\mathrm{sp}_{n}}<K_{\mathrm{eq}_{n}}$ (i.e, $\Omega_{n}<1.0$ ), the reaction proceeds from left to right (i.e., $\mathrm{NaCl}$ dissolves).

(3) At equilibrium, the $K_{\mathrm{sp}_{n}}$ equals $K_{\mathrm{eq}_{n}}$ (i.e, $\Omega_{n}=1.0$ ) and the saturation index is $\mathrm{SI}_{n}=$ $\log _{10}\left(\Omega_{n}\right)=0$.

Despite the large number of experimental studies, e.g., Fujimaki and Shimano (2006), Nachshon et al. (2011a,b), Rad and Shokri (2012), Rad Norouzi et al. (2013) and Norouzi Rad and Shokri (2014) looked into evaporative salinization in the shallow subsurface, to the best of our knowledge, only Jambhekar et al. (2015) investigated salt precipitation under nonisothermal conditions in a coupled FF-PM system. In this paper, we extend the REV-scale approach for evaporative salinization discussed in Jambhekar et al. (2015) to reactive transport and mixed salt precipitation. Here, we use a sophisticated approach for the description of mixed salt precipitation-dissolution processes.

\section{Model Concept}

The focus of this work is to couple the REV-scale single-phase compositional non-isothermal FF and the multi-phase compositional non-isothermal PM flow systems, for mixed salt precipitation near the FF-PM interface, as shown in Fig. 7. Here, in the FF subdomain Stokes flow is used, whereas in the PM subdomain Darcy's flow is assumed.

The basic coupling strategy to account for mass, momentum and energy exchange between the subdomain was presented in detail by Mosthaf et al. (2011), which is extended to shallow subsurface salinization by Jambhekar et al. (2015). The coupling conditions are based on the phenomenological explanations and are valid on the REV scale (Mosthaf et al. 2011; Baber et al. 2012). In this current model, formation of boundary layer at the FF-PM interface, flow separation and radiation are not included. For further details, we recommend (Mosthaf et al. 2011; Jambhekar et al. 2015). In the following, the PM submodel is summarized in Sect. 4.1, the FF model is discussed in Sect. 4.2, and the coupling conditions are elaborated in Sect. 4.3.

\subsection{Porous-Media Submodel}

The PM submodel is based on the following assumptions:

- Local thermodynamic (mechanical, thermal and chemical) equilibrium,

- Rigid solid phase (solid matrix and precipitated salt) (subscript S),

- Two-phase flow consisting of a liquid phase (subscript l) and a gas phase (subscript g), 


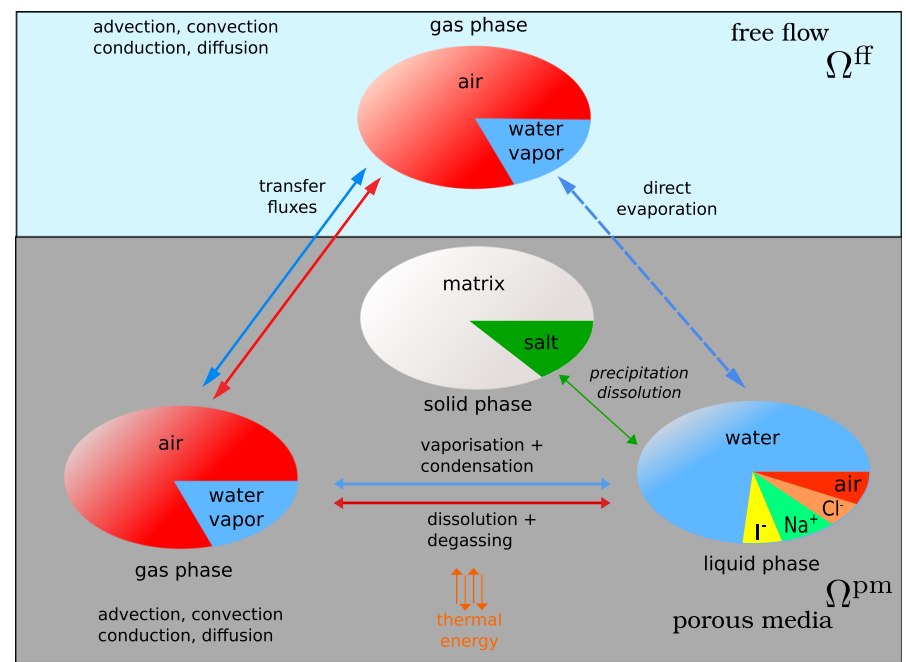

Fig. 7 Two fluid phases and two solid phases, namely solid sand matrix and solid salt, may be present in the porous medium subdomain $\Omega^{\mathrm{pm}}$, whereas the free-flow domain $\Omega^{\mathrm{ff}}$ is considered as single-phase region. The liquid phase consists of dissolved air and ionic species, e.g., $\mathrm{Na}^{+}, \mathrm{Cl}^{-}$and $\mathrm{I}^{-}$. The gas phase is composed of dry air and water vapor. There is no exchange of dissolved salt ions between liquid and gas phases. It is only exchanged with the solid phase through precipitation or dissolution. Both fluid phases in the porous medium interact with the gas phase in the free-flow subdomain (modified after Mosthaf et al. 2011; Jambhekar et al. 2015)

- Gas phase is assumed to be ideal,

- Slow flow velocities $(R e \ll 1)$ allow usage of the multi-phase Darcy's law,

- Binary diffusion is assumed in fluid phases, dispersion is neglected because of slow flow velocities and relatively high diffusion coefficient in the gas phase.

The new model allows exchange of components between phases and therefore is capable to describe evaporation and condensation, as well as dissolution and degassing processes. The definitions for the respective parameters, material laws and equations of state are presented in Table 2. Table 3 summarizes the employed conservation equations and chosen primary variables in the PM subdomain $\Omega^{\mathrm{pm}}$. It comprises of

- Two component mass balance equations for water $\mathrm{w}$ and dissolved salt $\mathrm{s}$, where $\phi$ is porosity, $\varrho_{\mathrm{mol}, \alpha}$ is the molar density for phase $\alpha, x_{\alpha}^{\kappa}$ is the mole fraction of component $\kappa$ in phase $\alpha, S_{\alpha}$ is the saturation of phase $\alpha, \mathbf{v}_{\alpha}$ is the velocity of phase $\alpha, D_{\alpha, \mathrm{pm}}^{\kappa}$ is the macroscopic diffusion coefficient for component $\kappa$ in phase $\alpha$, and $q^{k}$ is a source/sink term for the component. The source/sink for dissolved salt (s) is given by Eq. 12 .

- A mass balance equation for the gas phase $(\mathrm{g})$, where $\varrho_{\mathrm{g}}$ is the density of the gas.

- A mass conservation equation for precipitated solid salt, where $\phi_{\mathrm{S}}^{\mathrm{s}}$ is the pore volume fraction occupied by the precipitated solid salt.

- Based on the assumption of local thermal equilibrium one energy balance equation accounts for energy conservation. Here, $c_{\mathrm{S}}$ is the specific heat capacity of the solid phase, $c_{\mathrm{S}}^{\mathrm{S}}$ is the specific heat capacity of the solid salt, $u_{\alpha}$ is the internal energy of phase $\alpha, h_{\alpha}$ is the enthalpy of phase $\alpha, \lambda_{\mathrm{pm}}$ is the bulk thermal conductivity of the porous medium, and $\lambda_{\text {eff, },}$ and $\lambda_{\text {eff,g }}$ are the effective thermal conductivity of the liquid- and gas-saturated porous medium, respectively (see Table 2 ). 
Table 2 Material laws and equation of state dependent on primary variables

\begin{tabular}{|c|c|c|}
\hline Parameters & Equation of state & References \\
\hline \multicolumn{3}{|l|}{ Density } \\
\hline Gas density $\varrho_{\alpha}$ & $\varrho_{\alpha}=f\left(\varrho^{\kappa}, x_{\alpha}^{\kappa}, p_{\alpha}, T\right)$ & IAPWS (2009) \\
\hline Liquid density $\varrho_{1}$ & $\begin{aligned} \varrho_{1} & =\varrho_{\mathrm{w}}+1000 X_{1}^{\mathrm{s}}\left\{0.668+0.44 X_{1}^{\mathrm{s}}\right. \\
& +\left[300 p-2400 p X_{1}^{\mathrm{s}}+T(80\right. \\
& +3 T-3300 X_{1}^{\mathrm{s}}-13 p \\
& \left.\left.\left.+47 p X_{1}^{\mathrm{s}}\right)\right] \times 10^{-6}\right\}\end{aligned}$ & Batzle and Wang (1992) \\
\hline Component water $\varrho^{\mathrm{W}}$ & Incompressible fluid & Reid et al. (1987) \\
\hline Component air $\varrho^{\mathrm{a}}$ & Ideal gas & Reid et al. (1987) \\
\hline Salt $\mathrm{NaCl} \varrho_{\mathrm{S}}^{\mathrm{NaCl}}$ & $2165.0 \mathrm{~kg} / \mathrm{m}^{3}$ & \\
\hline Salt $\mathrm{NaI} \varrho_{\mathrm{S}}^{\mathrm{NaI}}$ & $3670.0 \mathrm{~kg} / \mathrm{m}^{3}$ & \\
\hline Diffusion coefficient $D_{\alpha, \mathrm{pm}}$ & $D_{\alpha, \mathrm{pm}}=\tau \phi S_{\alpha} D_{\alpha}$ & Millington and Quirk (1961) \\
\hline Tortuosity $\tau$ & $\tau=\frac{\left(\phi S_{\alpha}\right)^{7 / 3}}{\phi^{2}}$ & Millington and Quirk (1961) \\
\hline Capillary pressure $p_{\mathrm{c}}$ & van Genuchten model $p_{\mathrm{c}}=f\left(s_{1}\right)$ & Genuchten (1980) \\
\hline Relative permeability $k_{r \alpha}$ & van Genuchten model & Genuchten (1980) \\
\hline Eff. thermal conductivity $\lambda_{\mathrm{pm}}$ & $\lambda_{\mathrm{pm}}=\lambda_{\mathrm{eff}, \mathrm{g}}+\sqrt{S_{\mathrm{l}}}\left(\lambda_{\mathrm{eff}, 1}-\lambda_{\mathrm{eff}, \mathrm{g}}\right)$ & Somerton et al. (1974) \\
\hline Internal energy $u_{\alpha}$ & $u_{\alpha}=h_{\alpha}-p_{\alpha} / \varrho_{\alpha}$ & \\
\hline \multicolumn{3}{|l|}{ Enthalpy $h$} \\
\hline Gas phase & $h_{\mathrm{g}}=x_{\mathrm{g}}^{\mathrm{W}} h_{\mathrm{W}}+x_{\mathrm{g}}^{\mathrm{a}} h_{\mathrm{a}}$ & \\
\hline Liquid phase & $\begin{array}{r}h_{1}\left(p_{1}, T\right)=X_{1}^{\mathrm{W}} h^{\mathrm{W}}+X_{1}^{\mathrm{a}} h^{\mathrm{a}} \\
+X_{1}^{\mathrm{s}}\left(h^{\mathrm{s}}+\Delta h^{\mathrm{s}}\right)\end{array}$ & Michaelides (1981) \\
\hline Component air & $h^{\mathrm{a}}=1005(T-273.15 \mathrm{~K})$ & IAPWS (2009) \\
\hline Component water & $h^{\mathrm{W}}=f\left(p_{\alpha}, T\right)$ & IAPWS (2009) \\
\hline Component salt & $h^{\mathrm{s}}=f\left(p_{\alpha}, T\right)$ & Michaelides (1981) \\
\hline \multicolumn{3}{|l|}{ Secondary variables } \\
\hline Saturation & $S_{\mathrm{g}}=1-S_{1}$ & \\
\hline Phase pressure & $p_{\mathrm{g}}=p_{\mathrm{c}}+p_{\mathrm{l}}$ & Genuchten (1980) \\
\hline Mole fraction & $\sum_{\kappa} x_{\alpha}^{\kappa}=1$ & \\
\hline Air in liquid pahse & Henry's law: $x_{\mathrm{l}}^{\mathrm{a}}=p_{\mathrm{g}}^{\mathrm{a}} / H_{\mathrm{g} 1}^{\mathrm{a}}$ & \\
\hline Vapor in gas phase & $x_{\mathrm{g}}^{\mathrm{W}}=p_{\text {sat,Kelvin }}^{\mathrm{W}} / p_{\mathrm{g}}$ & \\
\hline Kelvin's equation & $\begin{array}{l}p_{\text {sat,Kelvin }}^{\mathrm{W}}= \\
\quad \exp \left(\frac{-\left(p_{\mathrm{c}}+\psi_{\mathrm{s}}\right)}{\varrho_{\mathrm{mol}, \mathrm{l}} R T}\right) p_{\mathrm{sat}}^{\mathrm{W}}(T)\end{array}$ & Kelly and Selker (2001) \\
\hline Osmotic potential & $\psi_{\mathrm{s}}=-R T \varrho_{\mathrm{mol}, 1} \ln \left(x_{1}^{\mathrm{W}}\right)$ & Kelly and Selker (2001) \\
\hline
\end{tabular}

Here, it is impotent to note that the equation for liquid density provided by Batzle and Wang (1992) was originally developed for brines containing $\mathrm{NaCl}$ as a major component. However, in this work, we use this formulation also for density description of solutions containing both $\mathrm{NaCl}$ and $\mathrm{NaI}$, where $X_{1}^{\mathrm{s}}$ is the total mass fraction of dissolved salts in the liquid phase

- The flow velocity within the porous medium is given by Darcy's law, where $\mathbf{K}$ is the intrinsic permeability tensor, $p_{\alpha}$ is the phase pressure, $k_{r \alpha}$ is the relative permeability, and $\mu_{\alpha}$ is the dynamic viscosity for phase $\alpha$. Moreover, changes in the intrinsic permeability of the porous medium caused by salt precipitation is taken into account using Kozeny- 
Table 3 Porous-media equations and associated primary variables

Balance equations

Primary variables

Mass balance for component $\kappa \in\left\{w, a, \mathrm{Na}^{+}, \mathrm{Cl}^{-}, \mathrm{I}^{-}\right\}$:

$\sum_{\alpha \in\{1, \mathrm{~g}\}} \frac{\partial\left(\phi \varrho_{\mathrm{mol}, \alpha} x_{\alpha}^{\kappa} S_{\alpha}\right)}{\partial t}+\nabla \cdot \mathbf{F}^{\kappa}=q^{\kappa}$, $S_{1}$ or $x_{\alpha}^{\kappa}$

$\mathbf{F}^{\kappa}=\sum_{\alpha \in\{l, g\}}\left(\varrho_{\mathrm{mol}, \alpha} \mathbf{v}_{\alpha} x_{\alpha}^{\kappa}-D_{\alpha, p m}^{\kappa} \varrho_{\mathrm{mol}, \alpha} \nabla x_{\alpha}^{\kappa}\right)$,

$q^{\kappa}=\left\{\begin{array}{lll}r_{n} & \forall & \kappa \in\left\{\mathrm{Na}^{+}, \mathrm{Cl}^{-}, \mathrm{I}^{-}\right\} \\ 0 & \text { else }\end{array}\right.$

Mass balance for gas phases

$\frac{\partial\left(\phi \varrho_{\mathrm{g}} S_{\mathrm{g}}\right)}{\partial t}+\nabla \cdot\left(\varrho_{\mathrm{g}} \mathbf{v}_{\mathrm{g}}\right)=q_{\mathrm{g}}$,

$p_{\mathrm{g}}$

Mass balance for solid salt

$\frac{\partial\left(\phi_{\mathrm{S}}^{\mathrm{s}} \varrho_{\mathrm{mol}, \mathrm{S}}^{\mathrm{s}}\right)}{\partial t}=\sum_{\kappa} q^{\kappa}$

Darcy's law

$\mathbf{v}_{\alpha}=-\frac{k_{r \alpha}}{\mu_{\alpha}} \mathbf{K}\left(\nabla p_{\alpha}-\varrho_{\alpha} \mathbf{g}\right)$

Energy balance

$$
\begin{aligned}
& \left(1-\phi_{0}\right) \frac{\partial\left(\varrho_{\mathrm{S}} c_{\mathrm{S}} T\right)}{\partial t}+\frac{\partial\left(\phi_{\mathrm{S}}^{\mathrm{s}} \varrho_{\mathrm{S}}^{\mathrm{s}} c_{\mathrm{S}}^{\mathrm{s}} T\right)}{\partial t} \\
& \quad+\sum_{\alpha \in\{1, \mathrm{~g}\}} \frac{\partial\left(\phi \varrho_{\alpha} u_{\alpha} S_{\alpha}\right)}{\partial t}+\nabla \cdot \mathbf{F}_{T}=q_{T}, \\
& \mathbf{F}_{T}=\sum_{\alpha \in\{1, \mathrm{~g}\}} \varrho_{\alpha} h_{\alpha} \mathbf{v}_{\alpha}-\lambda_{\mathrm{pm}} \nabla T
\end{aligned}
$$

Carman relationship and Leverett scaling is used to update corresponding changes in the capillary pressure (see Jambhekar et al. 2015 for details).

Extensive description of the PM model concept is given in Mosthaf et al. (2011) and Jambhekar et al. (2015). The chosen primary variables for the porous medium are given in Table 3. Using the relations given in Table 2, the secondary variables are determined as functions of the primary variables. Here, $p_{\mathrm{c}}$ is the capillary pressure, and $x_{\alpha}^{\kappa}$ is the mole fraction of component $\kappa$ in phase $\alpha$. If one phase disappears, the phase saturation $S_{\alpha}$ can not be used as a primary variable. As stated in Class et al. (2002), here, we perform a primary variable switch with the mole fraction $x_{\alpha}^{\kappa}$.

\subsection{Free-Flow Submodel}

The model in the free-flow subdomain is based on the following assumptions:

- laminar or creeping gas-phase (g) flow,

- gas phase is composed of components water (w) and air (a) (see Fig. 7).

- negligible inertia forces for low Reynolds numbers,

- binary diffusion in the gas phase.

The Stokes equation used for FF momentum description neglects the inertial effects and assumes gravity as the only external force. The conservation equations in the FF subdomain $\Omega^{\mathrm{ff}}$ are summarized in Table 4. It consist of a mass balance equation for the gas phase $(\mathrm{g})$, a mass balance equation for water vapor (w), a momentum balance equation and an energy 
Table 4 Free-flow equations and associated primary variables

Balance equations

Primary variables

Mass balance for water vapor (w)

$\frac{\partial\left(\varrho_{\mathrm{mol}, \mathrm{g}} x_{\mathrm{g}}^{\mathrm{W}}\right)}{\partial t}+\nabla \cdot \mathbf{F}^{\mathrm{W}}=q^{\mathrm{W}}$,

$\mathbf{F}^{\mathrm{W}}=\left(\varrho_{\mathrm{mol}, \mathrm{g}} \mathbf{v}_{\mathrm{g}} x_{\mathrm{g}}^{\mathrm{W}}-D_{\mathrm{g}}^{\mathrm{W}} \varrho_{\mathrm{mol}, \mathrm{g}} \nabla x_{\mathrm{g}}^{\mathrm{W}}\right)$

Mass balance for gas phases

$\frac{\partial\left(\varrho_{\mathrm{g}, \mathrm{mol}}\right)}{\partial t}+\nabla \cdot\left(\varrho_{\mathrm{g}} \mathbf{v}_{\mathrm{g}}\right)=q_{\mathrm{g}}$

$p_{\mathrm{g}}$

Momentum balance

$\frac{\partial\left(\varrho_{\mathrm{g}} \mathbf{v}_{\mathrm{g}}\right)}{\partial t}+\nabla \cdot\left[p_{\mathrm{g}} \mathbf{I}-\mu_{\mathrm{g}}\left(\nabla \mathbf{v}_{\mathrm{g}}+\nabla \mathbf{v}_{\mathrm{g}}^{T}\right)\right]=\varrho_{\mathrm{g}} \mathbf{g}$, $\mathbf{v}_{x}, \mathbf{v}_{y}$

Energy balance

$\frac{\partial\left(\varrho_{\mathrm{g}} u_{\mathrm{g}}\right)}{\partial t}+\nabla \cdot\left(\varrho_{\mathrm{g}} h_{\mathrm{g}} \mathbf{v}_{\mathrm{g}}-\lambda_{\mathrm{pm}} \nabla T\right)=q_{T}$

balance equation. Here, same notations as in the PM subdomain are used. The chosen primary variables are also given in Table 4.

\subsection{Coupling Conditions}

Suitable coupling conditions must be applied at the FF-PM interface to account for the underlying physics of the exchange process for mass, momentum and energy between the FF and the PM. Based on the phenomenological explanations, coupling conditions for compositional non-isothermal coupled FF-PM system are developed by Mosthaf et al. (2011). As Mosthaf et al. (2011) stated, these conditions are motivated from the pore-scale processes and are also valid on the REV scale. This is then extended by Jambhekar et al. (2015) to the salinization applications. For brevity we only list them in the following.

In specific, the mechanical equilibrium is given by

(1) Continuity of normal stresses resulting in feasible jump in the gas-phase pressure

$$
\mathbf{n} \cdot\left[\left(\left(p_{\mathrm{g}} \mathbf{I}-\mu_{\mathrm{g}}\left(\nabla \mathbf{v}_{\mathrm{g}}+\nabla \mathbf{v}_{\mathrm{g}}^{\mathrm{T}}\right)\right) \mathbf{n}\right)\right]^{\mathrm{ff}}=\left[p_{\mathrm{g}}\right]^{\mathrm{pm}} .
$$

(2) Continuity of the mass fluxes normal to the interface

$$
\left[\varrho_{\mathrm{g}} \mathbf{v}_{\mathrm{g}} \cdot \mathbf{n}\right]^{\mathrm{ff}}=-\left[\left(\varrho_{\mathrm{g}} \mathbf{v}_{\mathrm{g}}+\varrho_{\mathrm{l}} \mathbf{v}_{\mathrm{l}}\right) \cdot \mathbf{n}\right]^{\mathrm{pm}} .
$$

(3) Beavers-Joseph-Saffman condition for the tangential component of the FF velocity (Beavers and Joseph 1967; Saffman 1971)

$$
\begin{aligned}
& {\left[\left(v_{\mathrm{g}}+\frac{\sqrt{k_{i}}}{\alpha_{\mathrm{BJ}}}\left(\nabla v_{\mathrm{g}}+\nabla v_{\mathrm{g}}^{\mathrm{T}}\right) \mathbf{n}\right) \cdot \mathbf{t}_{i}\right]^{\mathrm{ff}}=0,} \\
& i \in\{1, \ldots, d-1\},
\end{aligned}
$$

where $k_{i}=\mathbf{t}_{\mathbf{i}} \cdot\left(\mathbf{K t}_{\mathbf{i}}\right)$ is the tangential component of the permeability tensor.

The conditions for thermal equilibrium at the FF-PM interface are derived by Alazmi and Vafai (2001) and Alazmi and Vafai (2002). This consists of 
(1) Continuity of temperature

$$
[T]^{\mathrm{ff}}=[T]^{\mathrm{pm}} .
$$

(2) Continuity of heat fluxes

$$
\begin{aligned}
& {\left[\left(\varrho_{\mathrm{g}} h_{\mathrm{g}} \mathbf{v}_{\mathrm{g}}-\lambda_{\mathrm{g}} \nabla T\right) \cdot \mathbf{n}\right]^{\mathrm{ff}}} \\
& \quad=-\left[\left(\varrho_{\mathrm{g}} h_{\mathrm{g}} \mathbf{v}_{\mathrm{g}}+\varrho_{1} h_{1} \mathbf{v}_{1}-\lambda_{\mathrm{pm}} \nabla T\right) \cdot \mathbf{n}\right]^{\mathrm{pm}} .
\end{aligned}
$$

The chemical equilibrium at the FF-PM interface is represented by

(1) Continuity of mole fractions

$$
\left[x_{\mathrm{g}}^{\kappa}\right]^{\mathrm{ff}}=\left[x_{\mathrm{g}}^{\kappa}\right]^{\mathrm{pm}}
$$

(2) Continuity of component flux across the interface for water (w) and air (a)

$$
\begin{aligned}
& {\left[\left(\varrho_{\mathrm{g}} \mathbf{v}_{\mathrm{g}} x_{\mathrm{g}}^{\kappa}-D_{\mathrm{g}} \varrho_{\mathrm{g}} \nabla x_{\mathrm{g}}^{\kappa}\right) \cdot \mathbf{n}\right]^{\mathrm{ff}}} \\
& =\left[\left(\varrho_{\mathrm{g}} \mathbf{v}_{\mathrm{g}} x_{\mathrm{g}}^{\kappa}-D_{\mathrm{g}, \mathrm{pm}} \varrho_{\mathrm{g}} \nabla x_{\mathrm{g}}^{\kappa}\right.\right. \\
& \left.\left.\quad+\varrho_{1} \mathbf{v}_{1} x_{1}^{\kappa}-D_{1, \mathrm{pm}} \varrho_{1} \nabla x_{1}^{\kappa}\right)\right]^{\mathrm{pm}}
\end{aligned}
$$

Based on the choice of the models in the subdomains, two of the three conditions from Eqs. 17 and 22 must be employed. In the scope of this work, we choose conservation of normal gas-phase mass flux across the interface (see Eq. 17) and conservation of component flux for water vapor (see Eq. 22). For further details on the FF model and the coupling conditions, we refer to Mosthaf et al. (2011) and Jambhekar et al. (2015).

\section{Numerical Model}

A vertex-centered finite-volume method is used for space, and implicit Euler method is used for time discretization (Baber et al. 2012). The primary variables for the FF and PM subdomain are presented in Tables 3 and 4, and the switch criteria for existence of different phases are discussed in detail by Class et al. (2002). The unknown mole fractions are determined as secondary variables using the primary variable-dependent constitutive relationships given in Table 2.

The developed coupled model concept is implemented in the numerical framework $\mathrm{DuMu}^{x}$, a free and open-source simulator for flow and transport processes in PM (Flemisch et al. 2011), which is based on the Distributed and Unified Numerics Environment (DUNE) (Bastian et al. 2008). The discritization and solution schemes used for this work are addressed in detail by Baber et al. (2012).

\section{Experimental Setup}

The experimental setup is shown in Fig. 8a, where the sand column of $0.011 \mathrm{~m}$ in diameter and $0.035 \mathrm{~m}$ in height is placed in the X-ray chamber. The details of the experimental setup can be studied in detail in Rad Norouzi et al. (2013) and Jambhekar et al. (2015). In the following, we briefly discuss the experiments carried out by Rad Norouzi et al. (2013). 
(a)

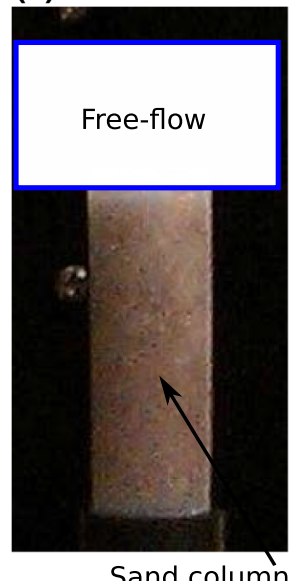

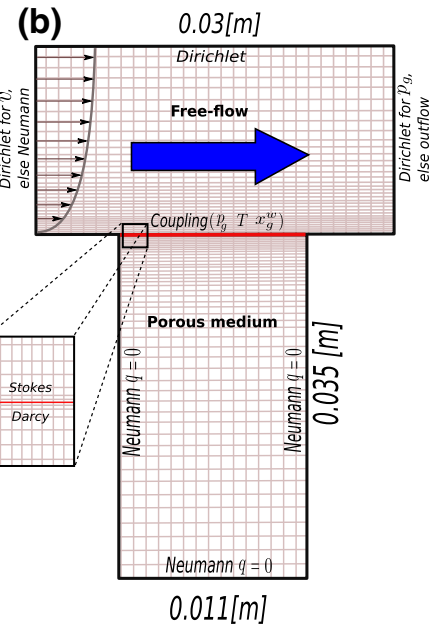

(c)

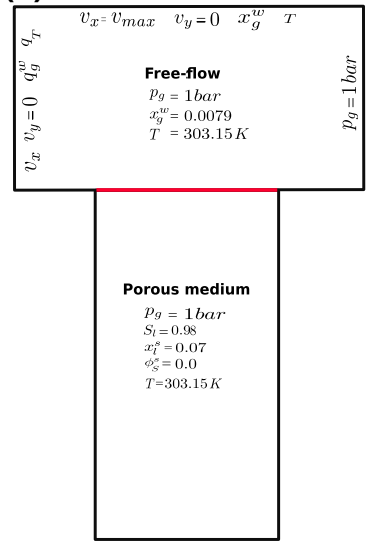

Fig. 8 a Setup for the experiments performed in an X-ray chamber at the University of Manchester to study stage SS1 evaporative salinization. Free-flow region of influence is shown on top of the sand column. $\mathbf{b}$ Simulation setup and schematic representation of grid. $\mathbf{c}$ Initial and boundary conditions

Table 5 Initial conditions for the porous medium and the free flow used for experiments

\begin{tabular}{ll}
\hline Primary variable & Initial conditions \\
\hline Free flow & \\
Velocity $\left(v_{\mathrm{g}}\right)$ & $0.01 \mathrm{~m} / \mathrm{s}$ \\
Humidity $\left(x_{\mathrm{g}}^{w}\right)$ & $0.007925(25 \%)$ \\
Temperature $(T)$ & $303.15 \mathrm{~K}$ \\
Porous medium & \\
Saturation $\left(S_{l}\right)$ & $98 \%$ \\
Salinity $(\mathrm{molal}(\mathrm{M}))$ & $3.5 \mathrm{~mol} / \mathrm{kg}$ \\
Solidity $\left(\phi_{\mathrm{S}}^{\mathrm{s}}\right)$ & $0.0(-)$ \\
\hline
\end{tabular}

Here, the sand column interacts with the FF only at its top surface, for which the recorded FF velocity, relative humidity and temperature in the X-ray chamber are given in Table 5. It also shows initial PM saturation, salinity and solidity. During the drying of the sand column, $\mathrm{X}$-ray scans were acquired for the duration of $19 \mathrm{~h}$ of stage SS1 evaporation. The cumulative mass loss of water and salt precipitation during this stage were determined from 3D image analysis. Above, stage SS1 refers to the stage in which evaporative salinization takes place at the free-flow-porous-media interface (for details, see Jambhekar et al. 2015).

Even though the evaporative demand was nearly constant during experiments, the relative humidity and temperature inside an X-ray chamber could not be controlled precisely (Rad Norouzi et al. 2013). So approximate temperature and relative humidity are used for numerical simulations. To investigate the dependence of the salinization behavior on initial salt concentration, three rounds of evaporation experiments were conducted in Rad Norouzi et al. (2013) for sand columns saturated with 3.5, 4.0 and 6.0 M saline water solution. 


\section{Simulation Setup}

The two-dimensional simulation setup given in Fig. 8b replicates the evaporating sand column in contact with the FF. The initial and boundary conditions for the PM subdomain are shown schematically in Fig. 8c and are summerized in Table 6. The van Genuchten model is used for capillary pressure-saturation relationship and relative permeability-saturation relationships. PM properties like porosity $\left(\phi_{0}\right)$, permeability $\left(K_{0}\right)$, solid thermal conductivity $\left(\lambda_{\mathrm{S}}\right)$ and specific heat capacity $\left(C_{\mathrm{p}}\right)$ and the Van Genuchten parameter $\alpha$ and $n$ are given in Table 7. Corresponding citations are also given in Table 7, and the values without citation are commonly used in the literature.

The FF subdomain $\Omega^{\mathrm{ff}}$ is chosen as $0.02 \times 0.03 \mathrm{~m}$ in size as shown in Fig. 8 b. A Dirichlet condition with parabolic shape is used for velocity at the left boundary, where maximum velocity $\left(\mathbf{v}_{\max }\right)$ is set to $0.01 \mathrm{~m} / \mathrm{s}$ at the top boundary and decreases to the Bravers-JosephSaffman slip velocity at the FF-PM interface. Neumann fluxes for energy and humidity at the inlet are determined using the inlet velocity

$$
\begin{aligned}
q_{\mathrm{g}}^{\mathrm{w}} & =\left(\mathbf{v}_{\mathrm{g}} \varrho_{\mathrm{mol}, \mathrm{g}} x_{\mathrm{g}, \mathrm{ref}}^{\mathrm{w}}\right) \cdot \mathbf{n}, \\
q_{\mathrm{T}} & =\left(\mathbf{v}_{\mathrm{g}} \varrho_{\mathrm{g}} h_{\mathrm{g}}\left(T_{\mathrm{ref}}\right)\right) \cdot \mathbf{n}
\end{aligned}
$$

where $x_{\mathrm{g}, \text { ref }}^{\mathrm{w}}$ and $T_{\text {ref }}$ are the initial conditions for mole fraction of water vapor in the gas phase and temperature. The initial, boundary and coupling conditions for the FF subdomain are shown schematically in Fig. 8c and are summerized in Table 6.

\section{Numerical Experiments}

In this section, the performance of the implemented model concept is tested by comparing the numerical results with the experimental data presented in Rad Norouzi et al. (2013). Here, one must notice that different from Jambhekar et al. (2015), as indicated in Table 3, in this work transport of dissolved ionic species is considered and precipitation-dissolution processes are accounted for by considering inter-ionic interactions.

In the following, both equilibrium and kinetic approaches for salt precipitation are applied, and the corresponding advantages and limitations are discussed. In Sect. 8.1, $\mathrm{NaCl}$ precipitation is analyzed using the equilibrium assumption, and in Sect. 8.2, the kinetic approach is employed. In Sect. 8.2, we also use inverse modeling for better prediction of the kinetic precipitation rate constant $k_{n}$ and fitting parameters $\theta$ and $\eta$ for $\mathrm{NaCl}$ precipitation. Finally, in Sect. 8.3 we analyze and discuss precipitation dynamics of $\mathrm{NaCl}$ and $\mathrm{NaI}$ in a mixed salt system.

\subsection{Equilibrium Approach}

Here, we apply the equilibrium approach for the numerical modeling and analysis of $\mathrm{NaCl}$ precipitation dynamics. The equilibrium condition implies immediate precipitation (i.e., $\dot{m}_{\mathrm{T}} \ll \dot{m}_{\mathrm{R}}$ ), as the dissolved salt concentration exceeds its solubility limit. For this approach, the dissolved salt precipitation $q^{\kappa} \forall \kappa \in\left\{\mathrm{Na}^{+}, \mathrm{Cl}^{-}\right\}$is given as

$$
q^{S}=\sum_{\kappa} q^{\kappa}=k_{\mathrm{p}} \phi S_{1} \varrho_{\mathrm{mol}, 1} \sum_{\kappa}\left|x_{\max , 1}^{\kappa}-x_{1}^{\kappa}\right|
$$




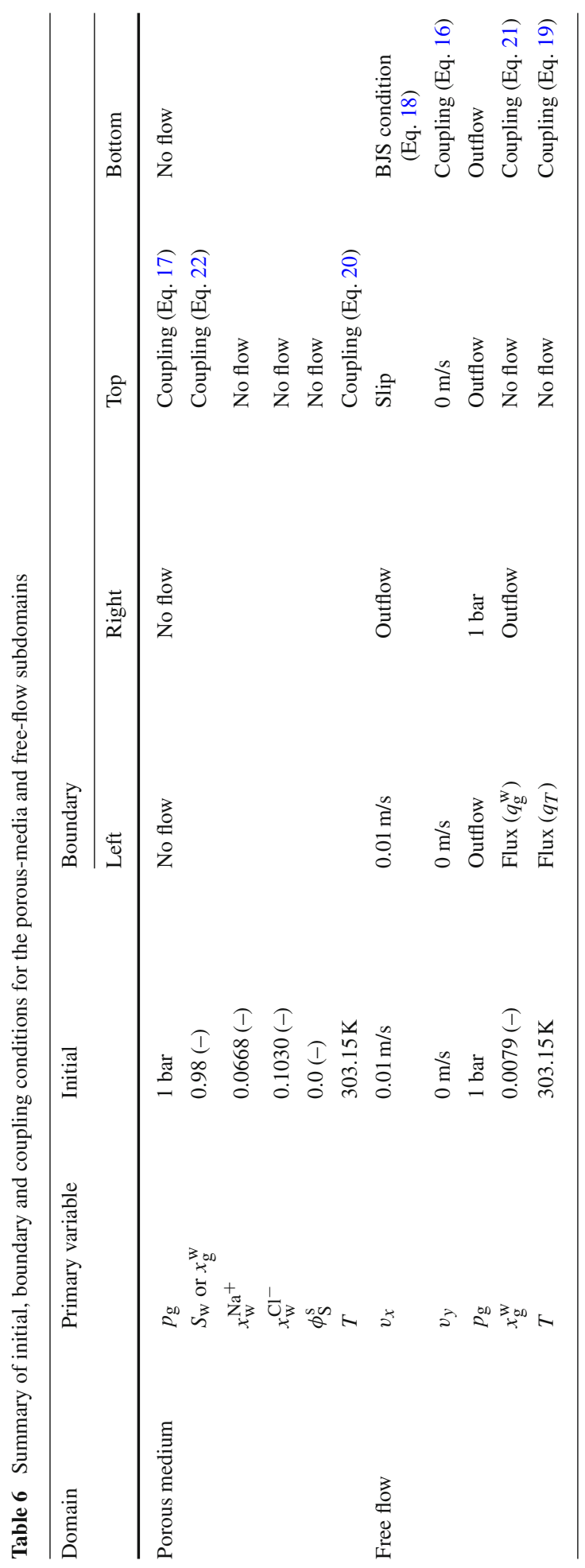


Table 7 Porous-media and free-flow properties for the reference experimental setup

\begin{tabular}{lll}
\hline Properties & Reference value & Unit \\
\hline Porous medium & & $(-)$ \\
Porosity $\left(\phi_{0}\right)$ & 0.37 & $\mathrm{~m}^{2}$ \\
Permeability $\left(K_{0}\right)$ (Shokri and Salvucci 2011$)$ & $6.37 \times 10^{-12}$ & $1 / \mathrm{Pa}$ \\
Van Genuchten $(\alpha)$ (Shokri and Salvucci 2011$)$ & $6.024 \times 10^{-4}$ & $(-)$ \\
Van Genuchten $(n)$ (Shokri and Salvucci 2011) & 12.18 & $\mathrm{~W} / \mathrm{mK}$ \\
Thermal conductivity solid matrix $\left(\lambda_{\mathrm{S}}\right)$ & 5.26 & $\mathrm{~J} / \mathrm{KgK}$ \\
Specific heat capacity solid matrix $\left(C_{\mathrm{p}}\right)$ & 830 & $\mathrm{Kgm}$ \\
Solid-salt density $\left(\varrho_{\mathrm{S}}^{\mathrm{S}}\right)$ & 2165 & $\mathrm{~J} / \mathrm{KgK}$ \\
Solid-salt specific heat capacity $\left(C_{\mathrm{p}, \mathrm{S}}\right)$ & 629 & $(-)$ \\
Free flow & & \\
Beaver-Joseph coefficient $\left(\alpha_{\mathrm{BJ}}\right)$ & 1.0 & \\
\hline
\end{tabular}

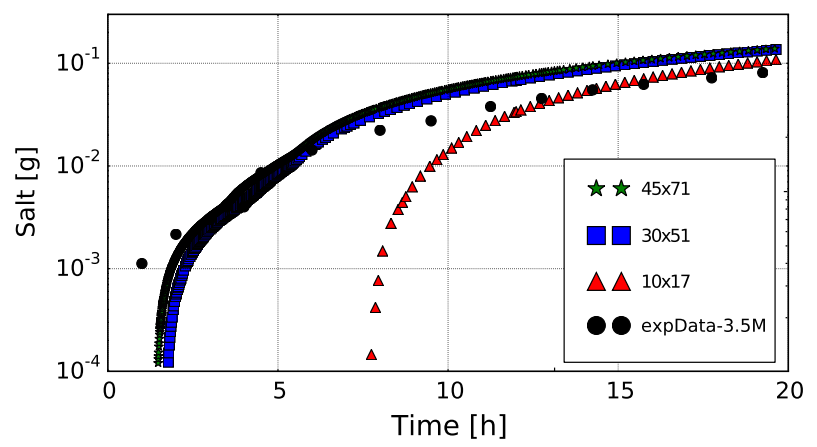

Fig. 9 Grid convergence analysis for the equilibrium salt precipitation approach during drying of 3.5 M saline sand column. Here, the grid resolutions of $10 \times 17$ (red), $30 \times 51$ (blue) and $45 \times 71$ (green) are used

where $k_{\mathrm{p}} 1 / \mathrm{s}$ is the rate constant and $x_{\max , 1}^{\kappa}$ refers to the solubility limit mole fraction of salt (i.e., for $\mathrm{NaCl} 6.12 \mathrm{M}$ at ambient conditions). The simulation setup is discussed in Sect. 7.

Figure 9 shows the grid convergence analysis for cumulative salt precipitation. In this work, we use three different grid configurations: in specific, $10 \times 17,30 \times 51$ and $45 \times 71$. The grid convergence analysis highlights that the simulation result for cumulative salt precipitation are grid independent for resolutions higher than $30 \times 51$. Therefore, for further simulations we use $30 \times 51$ grid.

Figure 10a, b displays the simulation results for cumulative mass loss of water and cumulative salt precipitation, respectively, for 3.5, 4.0 and 6.0 M solutions under identical FF and PM conditions. Here, the black dotted lines represent experimental observations and the colored plots are the simulation results. The comparison clearly points out that the simulated cumulative water loss and cumulative salt precipitation are convincingly in agreement with the experiments.

The differences between the simulation and experimental results are related to: (1) minor fluctuations in the FF temperature and humidity in the X-ray chamber during the experiments as reported by Rad Norouzi et al. (2013), which are not accounted by the current model concept, (2) marginal water ponding on top of the PM at the beginning of the experiment 


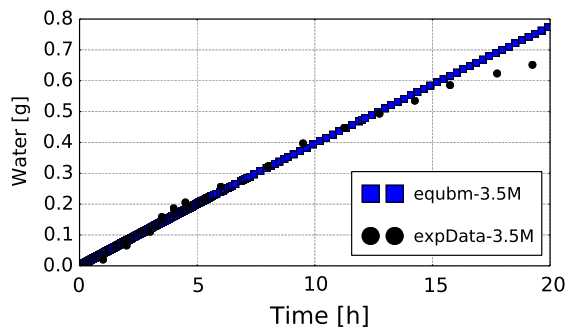

(1) 3.5 Molal

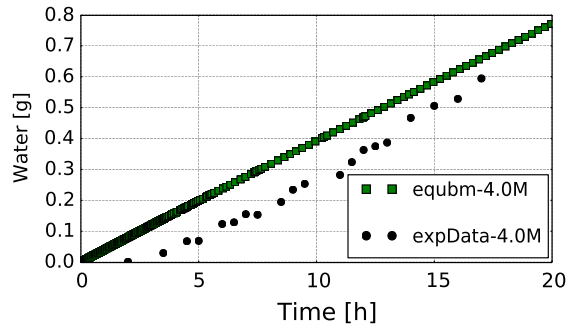

(2) 4.0 Molal

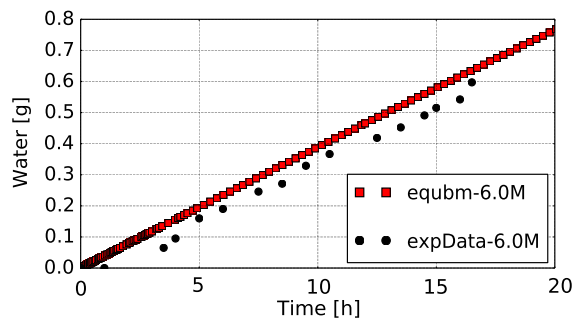

(3) 6.0 Molal

(a)

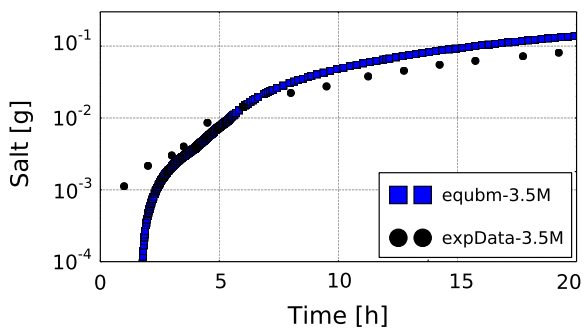

(1) 3.5 Molal

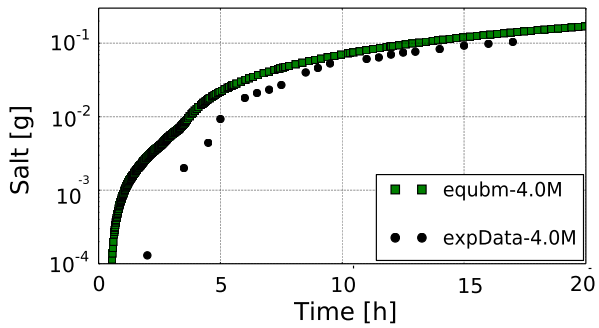

(2) 4.0 Molal

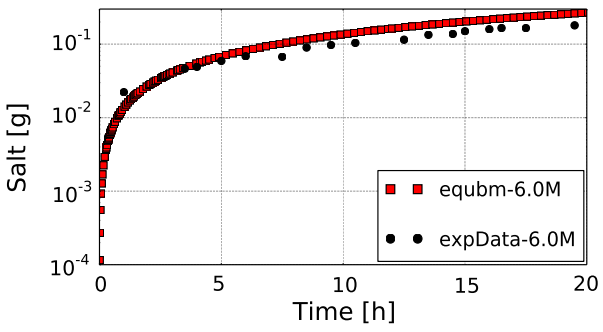

(3) 6.0 Molal

(b)

Fig. 10 Precipitation of single dissolved salt based on equilibrium assumption. Here, a the cumulative mass loss of water by evaporation from the saline sand columns, initially saturated with different concentrations of $\mathrm{NaCl}$ solution. The sand columns are subjected to identical FF and PM conditions. b Corresponding cumulative $\mathrm{NaCl}$ precipitation

which can not be explicitly considered during the numerical modeling, (3) inaccurate scaling of the PM intrinsic permeability and change in the capillary pressure by the Kozeny-Carman relationship, (4) experimental errors in the image analysis of the X-ray CT data. Furthermore, the simulated cumulative salt precipitation during early evaporation hours differs to a small extent from experiments, especially for the 3.5 and $4.0 \mathrm{M}$ cases. This is because of the preferential evaporation and salt precipitation in the fine pores on the microscale. Such microscale effects are, however, not captured on the volume averaged REV scale. Here, related to the averaged description, salt precipitation is first observed when the salt concentration for the complete REV approaches its solubility limit.

As discussed above, the reactive transport and equilibrium precipitation approach convincingly predicts the cumulative $\mathrm{NaCl}$ precipitation and cumulative water loss during evaporative salinization. Moreover, the simulation results presented above differ marginally from the equilibrium approach discussed in Jambhekar et al. (2015). These difference are related to the 
Table 8 Guess values for the fitting parameters of kinetic precipitation-dissolution model given by Eq. 12

\begin{tabular}{lllllll}
\hline Parameters & Guess1 & Guess2 & Guess3 & Guess4 & Guess5 & Guess6 \\
\hline$k_{n}\left(\mathrm{~mol} / \mathrm{m}^{2} \mathrm{~s}\right)$ & 1.0 & $1 \mathrm{e}-3$ & $6.5 \mathrm{e}-6$ & 1.0 & $1 \mathrm{e}-3$ & $6.5 \mathrm{e}-6$ \\
$\theta(-)$ & 1.0 & 1.0 & 1.0 & 1.0 & 1.0 & 1.0 \\
$\eta(-)$ & 1.0 & 1.0 & 1.0 & 1.3 & 1.3 & 1.3 \\
\hline
\end{tabular}

differences in the model concept and the conservation equations used in the porous-media subdomain models.

For the existence of multiple ionic species in a natural system, as discussed in Sect. 3.4, it is rather difficult to justify the assumption of equilibrium precipitation, and the generalized kinetic approach should be employed. Therefore, in the following, firstly, in Sect. 8.2, we employ and analyze the kinetic approach for $\mathrm{NaCl}$ precipitation dynamics, and later in Sect. 8.3, we apply it for the mixed $\mathrm{NaCl}-\mathrm{NaI}$ precipitation and also compare the results with equilibrium approach.

\subsection{Kinetic Approach}

Here, we use the kinetic approach given by Eq. 12 to model and analyze $\mathrm{NaCl}$ precipitation in a coupled FF-PM system. The simulation domain, discretization grid, initial condition and boundary conditions for the FF and the PM subdomains are identical to the simulation setup used in Sect. 8.1. For the kinetic precipitation approach, to the best of our knowledge, the precipitation kinetics is poorly characterized for many salts (here, $\mathrm{NaCl}$ ), and parameters such as precipitation rate constants $k_{n}$ and exponents $\theta$ and $\eta$ are not available at hand.

Therefore, in this section, we first calibrate the kinetic precipitation model against experimentally observed $\mathrm{NaCl}$ precipitation by Rad Norouzi et al. (2013) to find appropriate values of $k_{n}, \theta$ and $\eta$. In the following, numerical simulations are performed using some guess values for $k_{n}, \theta$ and $\eta$ given in Table 8 and inspired from the literature, e.g., Xu et al. (2012). As illustrated in Table 8, in this work, we use three different precipitation rate constants $k_{n}$ in combination with two values for the fitting parameter $\eta$. The value for parameter $\theta$ is kept unity.

Simulation results for the kinetic precipitation approach using different combinations of $k_{n}$ and $\eta$ are presented in Fig. 11. Figure 11a displays that the cumulative water loss for all the simulations is excellent in agreement with the experimental observations. Figure 11b points out that the salt precipitation dynamics is significantly dominated by the kinetic precipitation rate constant $k_{n}$, and moreover, the exponent $\eta$ influences the shape of the plot.

The comparison for cumulative salt precipitation in Fig. $11 \mathrm{~b}$ identifies that for that Guesses except Guess 3 and Guess 6 in Table 8, the numerical results are in excellent agreement with the experimental observations.

\subsection{Mixed Salt Precipitation}

In this section, we present a numerical case study, where we apply the developed kinetic model for mixed salt precipitation. The two-dimensional simulation setup for this case is shown in Fig. 12. The PM subdomain is $0.25 \times 0.25 \mathrm{~m}$ and the FF subdomain is $0.4 \times 0.2 \mathrm{~m}$. For grid convergence analysis, three different configurations, namely $15 \times 26,30 \times 51$ and $35 \times 61$, are used for the complete coupled FF-PM system with refinement at the FF-PM 


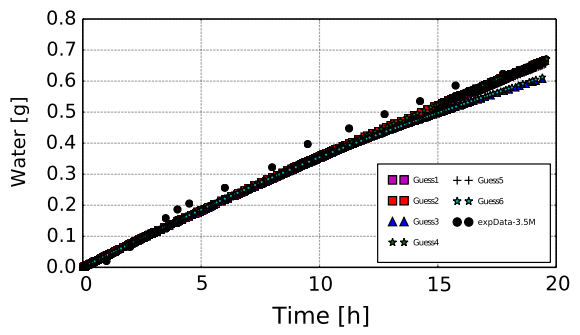

(1) 3.5 Molal

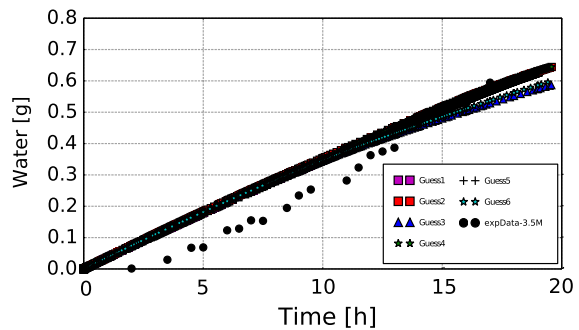

(2) 4.0 Molal

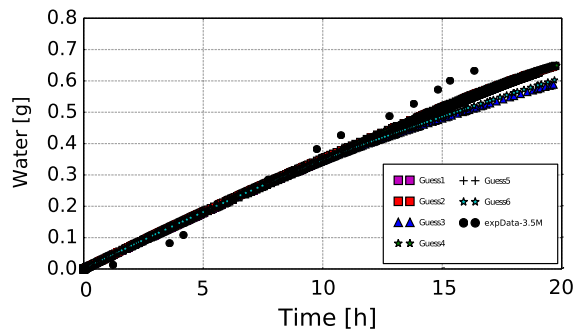

(3) 6.0 Molal

(a)

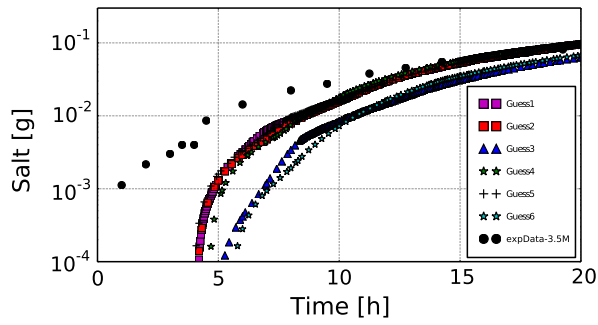

(1) 3.5 Molal

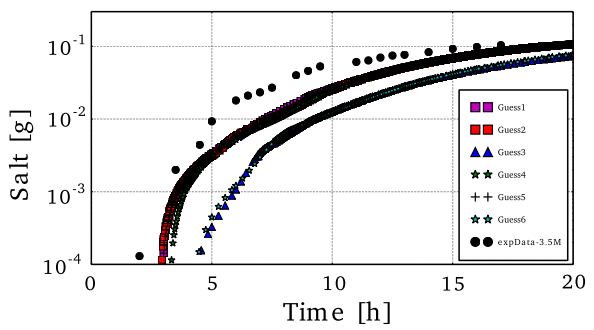

(2) 4.0 Molal

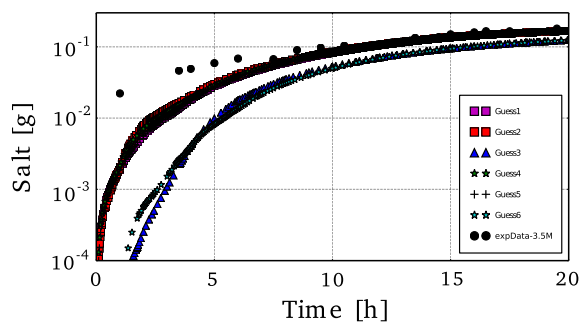

(3) 6.0 Molal

(b)

Fig. 11 Precipitation of single dissolved salt based on the kinetic approach. Here, a the cumulative mass loss of water by evaporation form the saline sand columns, initially saturated with different concentrations of $\mathrm{NaCl}$ solution. The sand columns are subjected to identical FF and PM conditions. b Corresponding cumulative $\mathrm{NaCl}$ precipitation

interface. The boundary conditions for the FF and PM subdomains and initial conditions for the FF subdomain are the same as in the earlier simulation setup, as discussed in Sect. 7. The initial conditions for the PM subdomain are discussed in the following.

Here, in the PM subdomain in addition to $\mathrm{Na}^{+}$and $\mathrm{Cl}^{-}$, reactive transport of ionic species $\mathrm{I}^{-}$is considered. Initially, the PM subdomain is assumed to be $98 \%$ saturated with saline water containing dissolved $\mathrm{NaCl}$ and $\mathrm{NaI}, 1.0 \mathrm{M}$ each; i.e., the initial condition of primary variables $\mathrm{Na}^{+}, \mathrm{Cl}^{-}$and $\mathrm{I}^{-}$is $2.0,1.0$ and $1.0 \mathrm{M}$, respectively. The initial condition for other primary variables in the PM subdomain are same as earlier simulation setup (see Sect. 7). In comparison with the earlier simulations in this case larger simulation domain and lower initial salt concentration are chosen: (1) to model evaporative salinization for longer durations and (2) to avoid complete clogging of the free-flow-porous-media interface due to precipitated salt. 


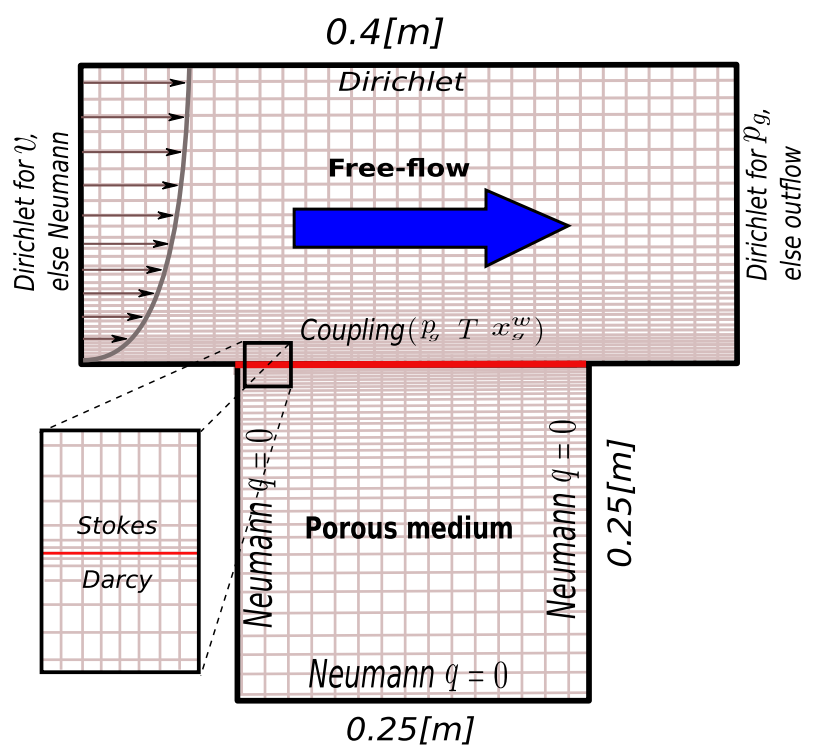

Fig. 12 Simulation setup for mixed salt precipitation in a coupled FF-PM system

Table 9 Guess values for the fitting parameters of kinetic precipitation-dissolution model (given by Eq. 12)

\begin{tabular}{ll}
\hline Parameters & $\mathrm{NaCl}-\mathrm{NaI}$ \\
\hline$k_{n}$ & $1 \mathrm{e}-3$ \\
$\theta$ & 1.0 \\
$\eta$ & 1.3 \\
\hline
\end{tabular}

The precipitation rate constant $k_{n}$ and the fitting parameters $\theta$ and $\eta$ for $\mathrm{NaCl}$ are taken from the earlier simulation study. Moreover, these parameters for $\mathrm{NaI}$ are not available at hand; therefore, as shown in Table 9, we start with the same parameter values as for $\mathrm{NaCl}$ precipitation. Numerical simulations are performed for 15 days of evaporative salinization. In the following, we study and analyze the numerical results for reactive transport of $\mathrm{Na}^{+}, \mathrm{Cl}^{-}$and $\mathrm{I}^{-}$and precipitation of $\mathrm{NaCl}$ and $\mathrm{NaI}$ under both equilibrium and kinetic conditions.

Figure 13 shows the grid convergence analysis for cumulative $\mathrm{NaCl}$ precipitation in a mixed salt system. Here, the grid convergence analysis highlights that the simulation result for cumulative salt precipitation are grid independent for resolutions higher than $30 \times 51$. Therefore, for further simulations we use $30 \times 51$ grid. Moreover, as depicted in Fig. 13, the cumulative $\mathrm{NaCl}$ precipitation for equilibrium precipitation approach also offers a very good in agreement with the kinetic approach.

During the course of evaporative salinization, the water vapor concentration in the ambient FF gas phase along the FF-PM interface increases due to water vapor exchange with the PM. Consequently, dissolved salt is transported toward the FF-PM interface and accumulates at the evaporation front. As a result, the activity of each ionic species, namely $\left[\mathrm{Na}^{+}\right],\left[\mathrm{Cl}^{-}\right]$and $\left[\mathrm{I}^{-}\right]$, increases at the evaporation front causing $\mathrm{NaCl}$ and $\mathrm{NaI}$ precipitation, provided that the kinetic saturation index for the corresponding salt species, i.e., $\Omega_{\mathrm{NaCl}}$ and/or $\Omega_{\mathrm{NaI}}$ exceeds unity. 


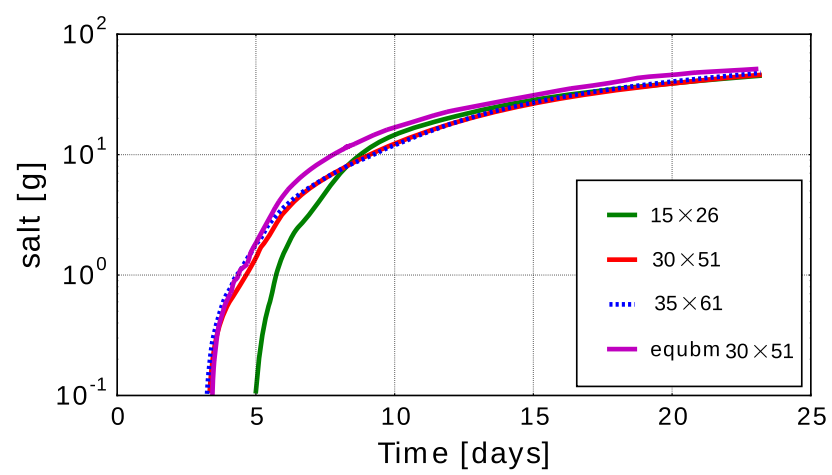

Fig. 13 Grid convergence analysis for the mixed salt precipitation approach during drying of saline sand column. Here, simulation results for cumulative $\mathrm{NaCl}$ precipitation are presented. For this analysis grid resolutions $15 \times 26$ (green), $30 \times 51$ (red) and $35 \times 61$ (blue) are used. In addition, mixed salt precipitation under equilibrium assumption is also illustrated for grid resolution $30 \times 51$ (magenta)

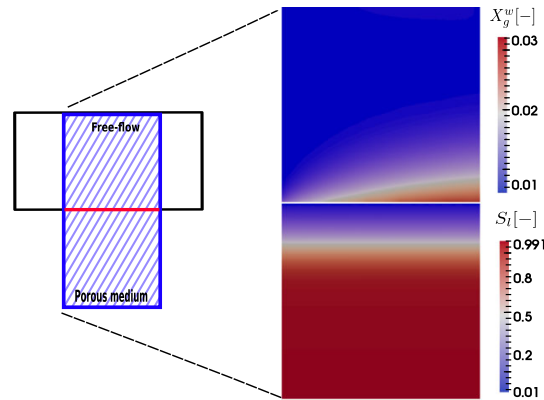

(a)

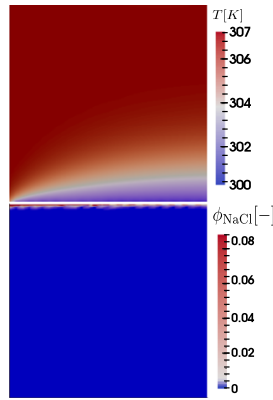

(b)

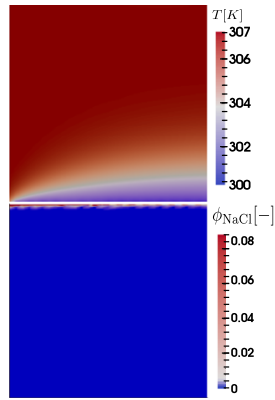

(c)

Fig. 14 Simulation results for a saline water saturation, b $\mathrm{NaCl}$ solidity $\left(\phi_{\mathrm{NaCl}}\right)$ and c $\mathrm{NaI}$ solidity $\left(\phi_{\mathrm{NaI}}\right)$ after 13 days of evaporative mixed salinization

Figure 14 shows simulation results for the saline water saturation distribution and the pore space occupied by precipitated $\mathrm{NaCl}$ and $\mathrm{NaI}$ after 13 days of evaporative mixed salt precipitation. Here, we present the simulation results in the region directly affected by the FF-PM interaction, as indicated by the striped region in Fig. 14a. Figure 14b, c points out that during evaporative salinization most of the salt precipitates near the FF-PM interface, which according to Nachshon et al. (2011a) and Jambhekar et al. (2015), is a typical feature of stage SS1 salinization. Here, for the given $k_{n}, \theta$ and $\eta$ in Table 9 and Fig. 14b, c indicate that the $\mathrm{NaI}$ solidity $\left(\phi_{\mathrm{NaI}}\right)$ is likewise the $\mathrm{NaCl}$ solidity $\left(\phi_{\mathrm{NaCl}}\right)$. This behavior is also consistently observed in Fig. 15a. Figure 15b displays the cumulative mass precipitation for $\mathrm{NaCl}$ and $\mathrm{NaI}$ during evaporative salinization. Here, related to the higher molal mass of $\mathrm{NaI}$, the cumulative mass of solid $\mathrm{NaI}>\mathrm{NaCl}$.

However, based on the phenomenological explanations discussed by Nachshon et al. (2011a), delay in the initiation of NaI precipitation is expected. This delay can be clearly seen in Fig. 15a, b, taking into account that same precipitation rate constant and exponents are used for these cases. In Fig. 16, the saturation indices $(\Omega)$ are plotted along a vertical section at the top of the PM subdomain after 1, 7 and 14 days. As shown in Fig. 16, early outset of 


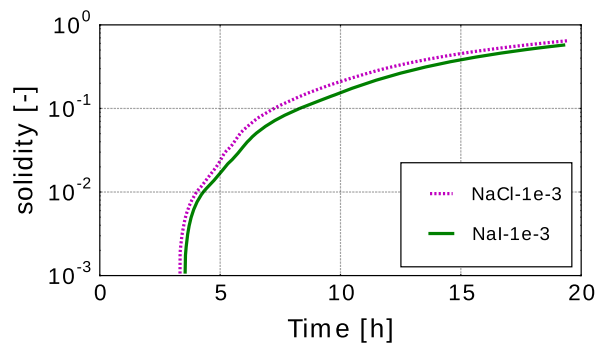

(a)

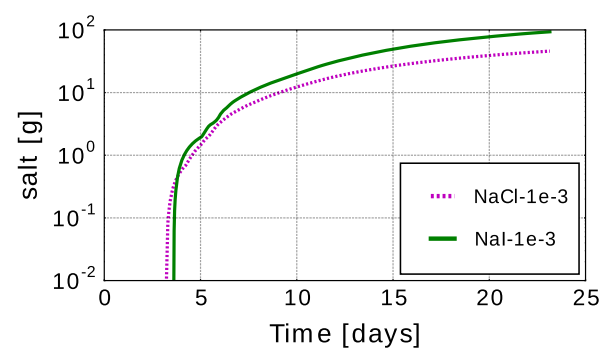

(b)

Fig. 15 a Cumulative solidity for precipitates of $\mathrm{NaCl}$ and $\mathrm{NaI}$ and $\mathbf{b}$ cumulative precipitation of salt, during evaporative salinization of a sand column exposed to ambient FF

Fig. 16 Comparison of the saturation index $\Omega$ for $\mathrm{NaCl}$ and $\mathrm{NaI}$ in the saline water-containing ionic species $\mathrm{Na}^{+}, \mathrm{Cl}^{-}$and $\mathrm{I}^{-}$. The values are plotted in the top $0.05 \mathrm{~m}$ of the PM subdomain. Here, the solid lines are the saturation index for $\mathrm{NaCl}$ $\left(\Omega_{\mathrm{NaCl}}\right)$, and the dotted lines are the saturation index for $\mathrm{NaI}$ $\left(\Omega_{\mathrm{NaI}}\right)$

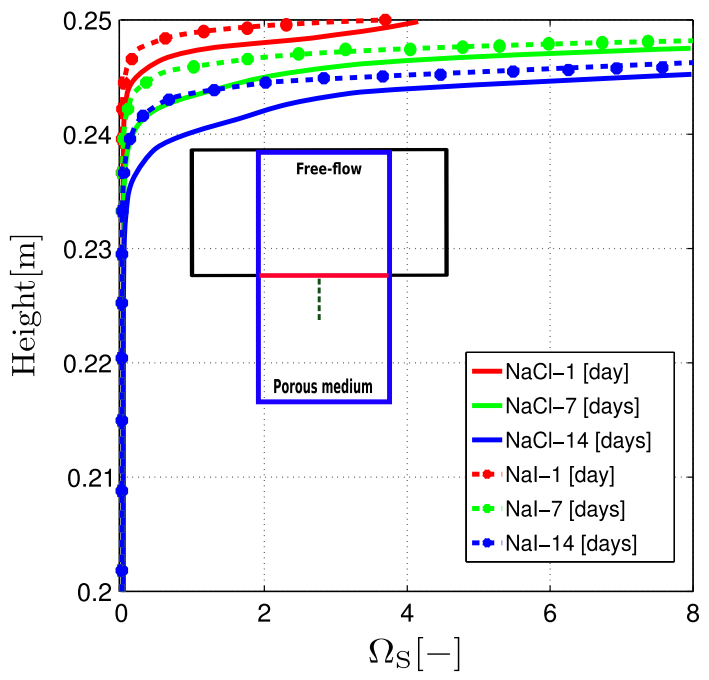

$\mathrm{NaCl}$ precipitation can also be predicted from the faster increase of $\Omega_{\mathrm{NaCl}}$ in comparison with $\Omega_{\mathrm{NaI}}$ locally along the PM depth.

Concentration distribution of dissolved ionic species in the top section $(0.2-0.25 \mathrm{M})$ of the PM after 1, 7 and 14 days of evaporation is shown in Fig. 17. Here, because of $\mathrm{NaCl}$ and NaI precipitation, significant decrease in the $\mathrm{Na}^{+}$and $\mathrm{Cl}^{-}$concentration is observed at the FF-PM interface. However, despite $\mathrm{NaI}$ precipitation, the $\mathrm{I}^{-}$concentration keeps increasing at the evaporation front. This indicates further potential $\mathrm{NaI}$ precipitation for longer drying durations.

From the above discussion, it is clear that the developed new model concept is capable to model ionic species transport and kinetic mixed salt precipitation in a coupled FF-PM system. For the mixed salt precipitation scenario, we initially assumed the same kinetic precipitation rate constant for $\mathrm{NaCl}$ and $\mathrm{NaI}$. In the following, we want to perform a sensitivity analysis of $\mathrm{NaI}$ precipitation dynamics in a mixed salt system to its precipitation rate constant $\left(k_{\mathrm{NaI}}\right)$.

Figure 18 exhibits the cumulative solidity evolution for kinetic NaI precipitation rates: $1 \mathrm{e}-3,1 \mathrm{e}-7$ and $1 \mathrm{e}-10$. Here, likewise precipitation behavior is observed for $k_{\mathrm{NaI}} 1 \mathrm{e}-3$ and $1 \mathrm{e}-7$. However, the precipitation dynamics largely changes for $k_{\mathrm{NaI}}=1 \mathrm{e}-10$. In this case, significant delay in NaI precipitation (approximately 1 day) is observed. 


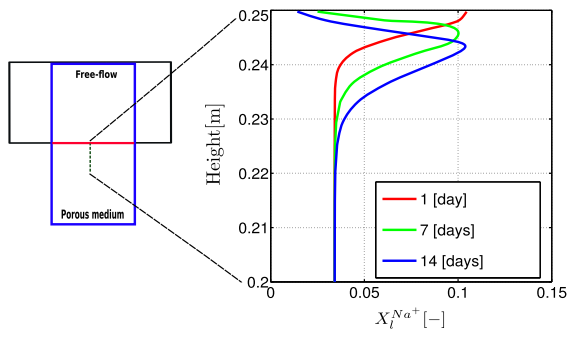

(a)

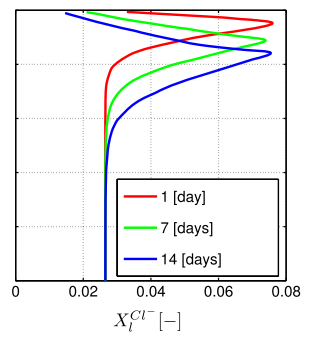

(b)

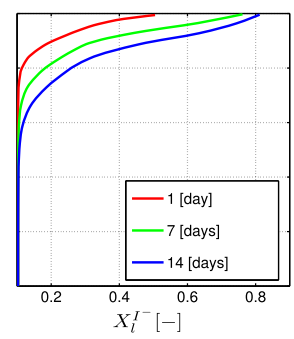

(c)

Fig. 17 Mass fraction distribution of different ionic species $\left(\mathbf{a ~ N a}{ }^{+}, \mathbf{b ~ C l}{ }^{+}, \mathbf{c ~ I}^{-}\right)$in the saline water along a vertical section in the top region $(0.2-0.25 \mathrm{~m})$ of the PM subdomain. The concentration distribution is plotted after 1,7 and 14 days for each ionic species

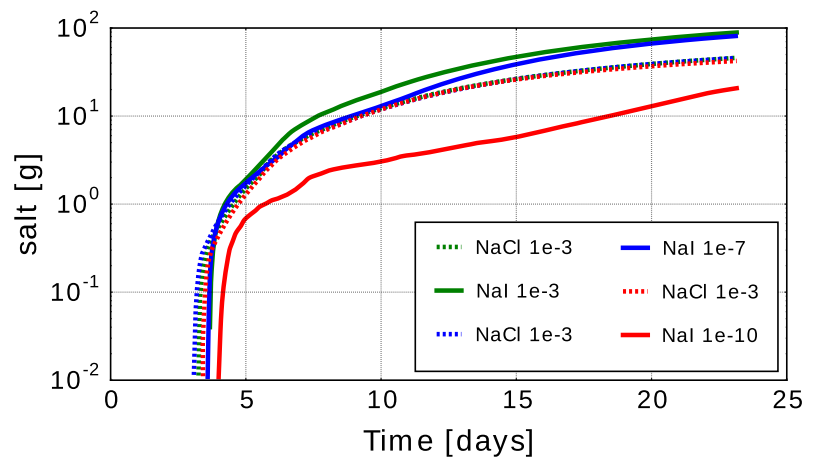

Fig. 18 Comparison of the solidity $\phi_{\mathrm{S}}$ of $\mathrm{NaCl}$ and $\mathrm{NaI}$ for different kinetic precipitation rate constants $\left(k_{\mathrm{NaI}}\right)$ for $\mathrm{NaI}$. Here, the kinetic precipitation rate constant $\left(k_{\mathrm{NaCl}}\right)$ for $\mathrm{NaCl}$ is kept constant

For a better prediction of the NaI precipitation rate constant, the new kinetic model must be validated against experiments. This rate constant can be further used in a mixed salt system, to acquire better understanding about the influence of inter-ionic interactions on $\mathrm{NaCl}$ and $\mathrm{NaI}$ precipitation dynamics. Furthermore, the delay in the initiation of $\mathrm{NaI}$ precipitation can also be validated by comparison against $\mathrm{NaCl}-\mathrm{NaI}$ precipitation experiments.

\section{Summary and Outlook}

A coupled FF-PM interaction model for evaporation-driven reactive transport and precipitation under both equilibrium and kinetic conditions is presented in the scope of this work. This new model is an extension of our previous work (Jambhekar et al. 2015). Non-isothermal compositional models are applied in the FF and PM subdomains and a simple interface, which cannot store mass, momentum and energy, is employed to account for the FF-PM interaction. The model is implemented in the open-source modeling framework DuMu${ }^{x}$ (Flemisch et al. 2011), where the vertex-centered-finite-volume (box) scheme is employed for spatial discretization and the implicit Euler method is used for time discretization.

The new concept is applied to model evaporative salinization under both equilibrium and kinetic conditions. As shown in Sect. 8.1, the numerical results for $\mathrm{NaCl}$ precipitation under equilibrium assumption are in accordance with experiments. However, for mixed salt 
precipitation in a natural system inter-ionic interactions between different salt ions play a significant role in the precipitation dynamics. Therefore, both equilibrium and generalized kinetic precipitation-dissolution approach are employed and the comparison is discussed.

As discussed in Sect. 8.2, the kinetic precipitation approach is in line with the experimental observations for $\mathrm{NaCl}$ precipitation. However, the kinetic precipitation rate constant $k_{n}$ and fitting parameters are usually not available at hand and must be determined explicitly for each salt type. Numerical simulations for $\mathrm{NaCl}-\mathrm{NaI}$ precipitation are observed to be analogous with the phenomenological explanations. However, for better understanding, model validation with $\mathrm{NaI}$ precipitation experiments must be undertaken in the near future. This should also help to better predict the mixed salt $(\mathrm{NaCl}-\mathrm{NaI})$ precipitation dynamics.

Due to constraint of space, in this work, we are unable to address combined precipitationdissolution processes in a mixed salt system. Salt dissolution processes in the shallow subsurface are rather complicated and are hardly addressed in the literature. Therefore, a detailed numerical modeling and analysis of salt dissolution processes are strongly recommended in the future.

The developed model concept can be applied to environmental, engineering and industrial applications, where mixed salt precipitation processes are significantly influenced by FF-PM interaction.

Acknowledgments This work is supported by the German research foundation (DFG) under the framework of the International research and training group NUPUS (GRK 1398). We thank Dr. Mansoureh Norouzi Rad, School of Chemical Engineering and Analytical Sciences, University of Manchester and Saideep Pavuluri, Institute of Petroleum Engineering, Heriot-Watt University, Edinburgh, for their valuable support. Dr. Nima Shokri acknowledges the funding by The Leverhulme Trust (RPG-2014-331) and the donors of the American Chemical Society Petroleum Research Fund for partial support of the experimental work (PRF No. 52054DNI6).

\section{References}

Alazmi, B., Vafai, K.: Analysis of fluid flow and heat transfer interfacial conditions between a porous medium and a fluid layer. Int. J. Heat Mass Transf. 44(9), 1735-1749 (2001). doi:10.1016/S0017-9310(00)002179

Alazmi, B., Vafai, K.: Constant wall heat flux boundary conditions in porous media under local thermal non-equilibrium conditions. Int. J. Heat Mass Transf. 45(15), 3071-3087 (2002). doi:10.1016/S00179310(02)00044-3

Appelo, C.A.J., Postma, D.: Geochemistry, Groundwater and Pollution, 2nd edn. Balkema, Leiden (2005)

Baber, K., Mosthaf, K., Flemisch, B., Helmig, R., Müthing, S., Wohlmuth, B.: Numerical scheme for coupling two-phase compositional porous-media flow and one-phase compositional free flow. IMA J. Appl. Math. 77(6), 887-909 (2012)

Bastian, P., Blatt, M., Dedner, A., Engwer, C., Klfkorn, R., Kornhuber, R., Ohlberger, M., Sander, O.: A generic grid interface for parallel and adaptive scientific computing. Part ii: implementation and tests in dune. Computing 82(2-3), 121-138 (2008). doi:10.1007/s00607-008-0004-9

Battistelli, A., Calore, C., Pruess, K.: The simulator tough2/ewasg for modelling geothermal reservoirs with brines and non-condensible gas. Geothermics 26(4), 437-464 (1997). doi:10.1016/S03756505(97)00007-2

Batzle, M.L., Wang, Z.: Seismic properties of pore fluids. Geophysics 57, 1396-1408 (1992). doi:10.1190/1. 1443207

Beavers, G.S., Joseph, D.D.: Boundary conditions at a naturally permeable wall. J. Fluid Mech. 30, 197-207 (1967). doi:10.1017/S0022112067001375

Class, H., Helmig, R., Bastian, P.: Numerical simulation of non-isothermal multiphase multicomponent processes in porous media.: 1. An efficient solution technique. Adv. Water Resour. 25(5), 533-550 (2002). doi:10.1016/S0309-1708(02)00014-3 
Clement, T.P., Hooker, B.S., Skeen, R.S.: Macroscopic models for predicting changes in saturated porous media properties caused by microbial growth. Ground Water 34(5), 934-942 (1996). doi:10.1111/j.1745-6584. 1996.tb02088.x

Crowe, A., Langstaffe, F.J.: Extension of geochemical modelling techniques to brine: coupling of the pitzer equations with models. In: Proceedings of Solving Groundwater Problems with Models. National Water Well Association Conference, Denver, CO, 10-12 Feb 1987

Domenico, P.A., Schwartz, F.W.: Physical and Chemical Hydrogeology. Wiley, New York (1990)

Flemisch, B., Darcis, M., Erbertseder, K., Faigle, B., Lauser, A., Mosthaf, K., Müthing, S., Nuske, P., Tatomir, A., Wolff, M., Helmig, R.: DuMuX: DUNE for multi-phase, component, scale, physics.., flow and transport in porous media. Adv. Water Resour. 34(9), 1102-1112 (2011). doi:10.1016/j.advwatres.2011. 03.007. New Computational Methods and Software Tools

Fujimaki, H., Shimano, T., Inoue, M., Nakane, K.: Effect of a salt crust on evaporation from a bare saline soil. Vadose Zone J 5(4), 1246-1256 (2006). doi:10.2136/vzj2005.0144

Gamazo, P., Saaltink, M., Carrera, J., Slooten, L., Bea, S.: A consistent compositional formulation for multiphase reactive transport where chemistry affects hydrodynamics. Adv. Water Resour. 35, 83-93 (2012). doi:10.1016/j.advwatres.2011.09.006

IAPWS: Revised release on the IAPWS formulation 1995 for the thermodynamic properties of ordinary water substance for general and scientific use. http://www.iapws.org (2009)

Jambhekar, V., Schröder, N., Helmig, R., Shokri, N.: Free-flow-porous-media coupling for evaporation-driven transport and precipitation of salt. Transp. Porous Med. 110, 251-280 (2015)

Kelly, S.F., Selker, J.S.: Osmotically driven water vapor transport in unsaturated soils. Soil Sci. Soc. Am 65, 1634-1641 (2001)

Lasaga, A.C., Soler, J.M., Ganor, J., Burch, T.E., Nagy, K.L.: Chemical weathering rate laws and global geochemical cycles. Geochim. Cosmochim. Acta 58(10), 2361-2386 (1994). doi:10.1016/00167037(94)90016-7

Mayer, K.U., Frind, E.O., Blowes, D.W.: Multicomponent reactive transport modeling in variably saturated porous media using a generalized formulation for kinetically controlled reactions. Water Resour. Res. 38(9), 13-1-13-21 (2002). doi:10.1029/2001WR000862

Michaelides, E.: Thermodynamic properties of geothermal fluids. Geotherm. Res. Counc. Trans. 5, 361-364 (1981)

Millington, R., Quirk, J.: Permeability of porous solids. Trans. Faraday Soc. 57, 1200-1207 (1961)

Mosthaf, K., Baber, K., Flemisch, B., Helmig, R., Leijnse, A., Rybak, I., Wohlmuth, B.: A coupling concept for two-phase compositional porous-medium and single-phase compositional free flow. Water Resour. Res. (2011). doi:10.1029/2011WR010685

Munns, D.R.: The Impact of Salinity Stress. CSIRO Division of Plant Industry, Canberra ACT (2011)

Nachshon, U., Shahraeeni, E., Or, D., Dragila, M., Weisbrod, N.: Infrared thermography of evaporative fluxes and dynamics of salt deposition on heterogeneous porous surfaces. Water Resour. Res. (2011). doi:10. 1029/2011WR010776

Nachshon, U., Weisbrod, N., Dragila, M.I., Grader, A.: Combined evaporation and salt precipitation in homogeneous and heterogeneous porous media. Water Resour. Res. (2011). doi:10.1029/2010WR009677

Norouzi Rad, M., Shokri, N.: Effects of grain angularity on nacl precipitation in porous media during evaporation. Water Resour. Res. 50(11), 9020-9030 (2014). doi:10.1002/2014WR016125

Pitzer, K., Kim, J.: Thermodynamics of electrolytes: 3 activity and osmotic corfficients for 2-2 electrolytes. J. Solut. Chem. 3, 539-546 (1974)

Rad, M.N., Shokri, N.: Nonlinear effects of salt concentrations on evaporation from porous media. Geophys. Res. Lett. (2012). doi:10.1029/2011GL050763

Rad Norouzi, M., Shokri, N., Sahimi, M.: Pore-scale dynamics of salt precipitation in drying porous media. Phys. Rev. E 88(32404), 1-5 (2013)

Reid, R.C., Prausnitz, J.M., Poling, B.E.: The Properties of Gases and Liquids. McGraw-Hill, Inc, New York (1987)

Saffman, R.: On the boundary condition at the surface of the Porous medium. Stud. Appl. Math. 50, 93-101 (1971)

Shokri, N., Salvucci, G.: Evaporation from porous media in the presence of a water table. Vadose Zone J 10(4), 1309-1318 (2011). doi:10.2136/vzj2011.0027

Somerton, W.H., El-Shaarani, A.H., Mobarak, S.M.: High temperature behavior of rocks associated with geothermal type reservoirs. In: SPE California Regional Meeting. Society of Petroleum Engineers, San Francisco, CA (1974). doi:10.2118/4897-MS

Steefel, C.I., Cappellen, P.V.: A new kinetic approach to modeling water-rock interaction: the role of nucleation, precursors, and Ostwald ripening. Geochim. Cosmochim. Acta 54(10), 2657-2677 (1990). doi:10.1016/ 0016-7037(90)90003-4 
Truesdell, A., Jones, B.: A Computer Program for Calculating Chemical Equilibrium of Natural Waters, vol. 73. U.S. Geological Survey, Washington, DC (1973)

Van Genuchten, M.T.: A closed-from equation for predicting the hydraulic conductivity of unstructured soil. Soil Sci. Soc. Am. J. 44, 892-898 (1980)

Xu, T., Spycher, N., Sonnenthal, E., Zheng, L., Pruess, K.: TOUGHREACT User's Guide: A Simulation Program for Non-Isothermal Multiphase Reactive Geochemical Transport in Variably Saturated Geologic Media, Version 2.0. Lawrence Berkeley National Laboratory, Berkeley, California, lbnl-draft edn. (2012) 